\title{
An improved localization scheme based on IMDV-hop for large-scale wireless mobile sensor aquaculture networks
}

\author{
Jianping Zhu' ${ }^{*}$, Chunfeng LV and Zhengsu Tao ${ }^{2}$
}

\begin{abstract}
With the hasteful development of ocean economy and the increasing exploiture of ocean resources, offshore water is contaminated seriously. Ocean ecological environment is unprecedentedly faced to threat and destruction. Moreover, the desire for aquatic and marine products is increasing consumedly according to people's health attention rising. It is extremely urgent to establish and maintain low-cost and high-efficient transmission and localization schemes for real-time large-scale aquaculture surveillance systems. Localization scheme IMDV-hop (Intermittent Mobile DV-hop) embedded in WLS (weighted least square) method, accompanying with HTC (Hidden Terminal Couple), is proposed in this work for the purpose of environment surveillance, object localization for early warning, rescue operations, and restructuring plan, etc. Two critical parameters, correction coefficient $k_{c}$ and weighted coefficient $w_{N_{x}, r}$ are introduced into IMDV-hop scheme for large-scale aquaculture monitoring and localization mobile sensor systems to evaluate the influence on localization behaviors, and subsequently guarantee localization accuracy and time-critical performance. And localization error, delay, and consumption are predicted by comprehensive NS-2 simulations. Besides, performance comparisons of IMDV-hop scheme with other DV-hop-based schemes and MCL-based scheme are also proposed. Analysis and comparison results show that delay behavior of IMDV-hop is improved largely relative to other schemes, while accuracy and energy consumption performance is improved in some cases of more node density and lower mobile velocity.
\end{abstract}

Keywords: DV-hop, Wireless sensor networks, IMDV-hop, WLS, HTC

\section{Introduction}

In recent years, wireless sensor networks (WSNs) have revolutionized the world of distributed systems and enabled many new applications. WSNs play more and more decisive roles in various aspects such as environmental and habitat monitoring, precision agriculture, animal tracking, disaster rescue and almost touch upon all aspects of our life. In many applications, it is essential for nodes to know their position information [1-4], that is, measurement data or information exchanges happened in WSNs without location information are meaningless. For example, locations must be known in environmental monitoring applications such as bush fire surveillance, water quality monitoring, and precision agriculture. And sensor positions can also help to facilitate the network with an

\footnotetext{
* Correspondence: jp-zhu@shou.edu.cn

'SOU College of Engineering Science and Technology, Shanghai Ocean University, No.999, Huchenghuan Rd, Shanghai, People's Republic of China Full list of author information is available at the end of the article
}

overall point of view, such as routing or connectivity for WSNs. Therefore, localizations of sensors or events have become fundamental elements in WSNs.

And also, growing close attentions are paid to underwater WSNs (UWSNs) [5, 6] during the last couple of years. Ocean explorations and a multitude of underwater applications such as oceanographic data collection, warning systems for natural disasters, ecological applications, military under water surveillance, and industrial applications are provoking people to focus on UWSNs. And people's growing needs for aquatic organisms to be rich in proteinic substance and other trace elements can be fulfilled or relieved through aquaculture farm. Our aquaculture farm cultivates the Chinese soft-shell turtle, which is a kind of precious and costly aquatic product for farmers. Turtles are equipped sensor nodes for farmers to obtain real-time positions of them and, subsequently, are executed on-time nurses if they encounter disease or inactivity, to increase survival ratio. And also, turtle positions can also be used for 
routing and networking purposes in such a large-scale surveillance WSNs. Of course, the straightforward method for localization in WSNs is to use Global Positioning System (GPS) [7], attached on every sensor node. However, it is infeasible for large-scale aquaculture monitoring WSNs equipped GPS receiver on every node, which brings about so much high cost or inconvenience.

Depending on whether absolute range measurements are used or not, localization schemes can be roughly classified into two categories [1-4]: range-based and range-free. Range-based algorithms measure the exact distance or angle of pending localization nodes adopting techniques such as TOA (time of arrival) $[8,9]$, TDOA (time difference of arrival) [10], RSSI (received signal strength indicator) [11-14], or AOA (angle of arrival) [15].

Acknowledging that high cost of hardware required by range-based solutions may be inappropriate in relation to the required location precision, researchers have sought alternate range-free solutions to localization problems in WSNs. Typical range-free algorithms [16-19] include centroid [20-22], CPE (convex position estimation) [23], APIT (Approximate Point in Triangle) [18, 24], and DV-hop (Distance Vector-hop) [25, 26]. Among range-free localization methods, centroid and CPE are relatively simple, having low complexity, but they require a normal node to have at least three neighboring anchors. DV-hop algorithm can handle the case where a normal node has less than three neighbor anchors, and computation complexity is relatively low which saves lots of energy consumption. Considering these interesting and attractive advantages of DV-hop algorithm, we prefer to localization methods based on DV-hop algorithm in our aquaculture WSNs.

The rest of this paper is structured as follows: Section 2 gives a summary of related works and analysis premise of our model. A novel localization algorithm IMDV-hop is proposed in Section 3, after presenting the inferiors of original DV-hop algorithm. Performance analysis models of IMDV-hop accompanying with HTC scheme are also presented in Section 3. In Section 4, accurate analyses and validations of localization error, localization delay, and energy consumption are presented using NS-2 simulator, and performance comparisons of our model with other models are also proposed. Finally, concluding remarks and future work are presented in Section 5.

\section{Related works}

Literature reviews presented here are twofold: (1) references related to localization schemes based on DV-hop algorithm for WSNs and (2) references related to localization schemes for mobile WSNs.

\subsection{DV-hop localization schemes}

DV-hop scheme is an attractive and low-energy consumption localization scheme, which is the most general range-free localization scheme, basing on connectivity information between nodes. Many algorithms based on DV-hop have been proposed these years. Not similar to other range-free localization schemes, DV-hop algorithm can handle the case where a node has less three neighbor anchors. Hence, for computation simplicity and sustainable localization accuracy of DV-hop scheme, our work focuses on DV-hop-based scheme.

Three steps, localization information exchange phase for obtaining hop counters, average hop distance computation phase for every anchor, and estimated position phase using trilateration or maximum likelihood estimation method, are firstly proposed by Niculescu and Nath in $[25,26]$. An improved DV-hop algorithm is proposed in [27] to reduce location error accumulated over multiple hops by using a differential error correction scheme. Difference or error between estimated distance of two anchors and actual distance of these anchors is calculated, and this error can be generalized to calculate estimated error of distance between unknown node and its nearest anchor. DV-Loc algorithm is proposed in [28], which is designed by use of Voronoi diagrams to limit the scope of flooding and error of computed positions to improve localization accuracy, that is, through improving accuracy of hop count. Anchors are previously divided into several levels. A Voronoi diagram is built based on position information of the first-level anchors firstly. Then, the second-level anchors compute average size of a hop like DV-hop. Other levels of anchors repeated these two steps until all levels of anchors are used. DDV-hop [29] and RDV-hop [30] localization schemes are proposed based on DV-hop algorithm, using a weighting method to determine a weighted distance-per-hop value for each normal node, which consumes additional energy for obtaining differential error or network topology separately. An advanced DV-hop (ADV-hop) localization scheme [31] uses the hop size of anchors, from which unknown node measures distance between anchors or between unknown node and anchors. Inherent error in estimated distance between anchor and unknown node is reduced in the third step of ADV-hop, and WLS algorithm is used, in which weight factor is set as the inverse of the minimum number of hops between unknown node and an anchor. And locations are refined by using extraneous information obtained by solving mathematical equations. A threshold $M$ is introduced in [32], which uses weighted average hop distance of anchors within $M$ hops, not all anchors in networks of original DV-hop scheme, to calculate average hop distance of unknown nodes, and location results are corrected of this DV-hop based scheme. HDV-hop (hybrid DV-hop) is proposed in [33] to obtain high localization accuracy and minimize flooding, and then reduce energy consumption, in which anchors are deployed only on the perimeter of WSN and not inside it. In the first step of 
HDV-hop, sensor $S$ maintains Hop Count $_{i}$ in a table called the HopCountsTable, and $S$ maintains ID of one-hop neighbor from which it has received the minimum HopCount value, referring to this minimum value as GlobalMinimum, the neighbor that delivered this value to $S$ as GlobalMinimumNeighbor. In the second step of HDV-hop, energy-expensive flooding phase is eliminated and each anchor sends its calculated average hop length to the base station via the RingofAnchors. A trilateration algorithm is executed by a powerful base station instead of individual sensors, and an iterative method to solve NLLS problem. Because of anchors are located on the perimeter of network, unknown node can consume lots of energy to establish or maintain DV-hop calculation chains.

Two refined localization algorithms, that is, hyperbolic-DV-hop localization algorithm and improved weighted centroid DV-hop localization scheme (IWC-DV-hop) are proposed in [34]. Instead of taking average hop size of the anchor nearest to unknown node, hyperbolic-DV-hop scheme chooses average hop sizes among all anchors as average hop size of unknown node. And also, selecting appropriate anchors and centroid scheme instead of maximum likelihood estimation scheme can improve accuracy for IWC-DV-hop algorithm.

Quad DV-hop and other two DV-hop-based schemes, iDV-hop1 and iDV-hop2, are proposed in [35] to improve localization accuracy. Quad DV-hop formulates localization problems as bounded least squares problems, to be solved by quadratic programming. Checkout DV-hop scheme and selected-3-anchor DV-hop are proposed in [36]. The former one adjusts position of a normal node based on its distance to the nearest anchor neighbor, in which a checkout step is added to change estimated position from $N_{D V-}$ hop to a new one called $N_{\text {Checkout }}$, a relative accuracy value for computing the distance between unknown node and each anchor. The other one chooses the best three anchors based on connectivity parameters. Mostly, three anchors can sufficiently localize a normal sensor, rather than involving in all available anchors in the network. But how to choose appropriate three anchors to improve localization accuracy requires taking network topology into account. Especially, non-slotted CSMA/CA scheme are embedded in Checkout DV-hop scheme and selected-3-anchor scheme to solve problems of frame collisions and link congestion, which however frequently happen during the broadcast of position frames and distance-per-hop frames, almost not considered in original DV-hop algorithm or other DV-hop-based schemes. Sub-square weighted (SSW) DV-hop algorithm is proposed and applied with rectangular topology in O-WSN (hybrid optical WSN) [37], and various factors that affect localization accuracy of DV-hop-based algorithm in WSNs are investigated, including communication radius of a node, number of beacon nodes, and number of total nodes.
Most DV-hop-based schemes are designed for localizing static WSNs. But DV-hop-based localization scheme can also be used for mobile WSNs such as selected-3-anchor scheme in [36], and we can adopt DV-hop-based scheme to mobile networks in this work. Mobility behaviors of DV-hop-based scheme are then analyzed elaborately, and localization performance such as error and delay is also compared with MCL (Monte Carlo localization)-based scheme. Impacts of mobility models on DV-hop-based localization in mobile WSNs are presented in [38].

Moreover, there are many measurement means for DV-hop-based localization schemes for WSNs. In the third step of the original DV-hop scheme, calculation of estimated location is achieved using trilateration method or maximum likelihood estimation method. Most of the localization schemes based on DV-hop algorithm adopt these two calculation methods, such as [36]. And normal sensor computes hop size based on all hop size values it receives from anchors, instead of just taking the first received hop size value, that is, hop size value of the nearest anchor in [38]. So, positions of normal nodes can be calculated by using WLS method. As related above, ADV-hop in [31] also uses WLS algorithm to calculate positions of unknown nodes. And also, hyperbolic location algorithm is also used to obtain locations of normal nodes related in [34,39].

\subsection{Localization schemes for mobile WSNs}

Most of the schemes related above focus on the localization for static sensor networks. However, mobile sensors are inevitably required in some applications such that target tracking, floats for sea environmental monitoring. Recently, many localization schemes are proposed for mobile sensor networks, and most of these algorithms are usually based on MCL method [40]. MCL algorithm has been extensively used in robotics [41], in which a robot estimates its location based on its motion, perception, and possibly a pre-learned map of its environment. Each step of MCL algorithm is divided into three steps: an initialization phase, a prediction phase, and an update phase. A node makes a movement and uncertainty of its position increases in prediction phase, and new measurements are incorporated to filter and update data in update phase. SMC (sequential MCL) algorithm is firstly used for mobile sensor networks in [42], without a predefined map or special hardware, assuming that time is divided into discrete time units. Localization process of SMC for WSNs is briefly related as follows. Each node localizes itself in each time interval. A sensor randomly chooses a set of $N$ samples $L_{0}=\left\{l_{0}^{0}, l_{0}^{1}, \ldots, l_{0}^{N-1}\right\}$, treating as localizations, within deployment area during localization initial phase. Then, SMC algorithm executes two steps to locate unknown nodes, the same as algorithm of MCL, prediction and filtering. A sensor generates a new set of localizations $L_{t}$ based on previous set $L_{t-1}$ at random 
time $t$ during the prediction. Random location $l_{t}^{i}$ is randomly chosen from the disk of radius $v_{\max }$ round $l_{t-1}^{i}$ on the premise of given location $l_{t-1}^{i}$ from $L_{t-1}$, and $v_{\max }$ is the maximum speed of nodes. During filtering phase, all impossible locations $l_{t}^{i}$ are deleted among new localizations $L_{t}$, using position information obtained from both one-hop and two-hop anchors. If a node cannot hear from its neighbor anchors, or not enough samples can be obtained, or too samples are neglected, localization error can be introduced in SMC. MCB (Monte Carlo localization boxed) is proposed in [42] to improve energy behaviors in SMC, in which drawing samples can easily swallow up lots of energy. In MCB scheme, to filter redundant samples, a node that heard one-hop or two-hop neighbor anchors builds a box which covers the region where anchors' radio range overlap. Location of a node can be not only constrained from anchors within one-hop and two-hop but also from its one-hop normal nodes in [43], rather than localization information is constrained only from anchors within one-hop or two-hop, such as SMC schemes related above. And also, impossible position samples are filtered out, through predicting moving direction, in which three mobility models are introduced in IMCL (improved MCL) scheme. An energy-efficient algorithm WMCL (weighted MCL), a further reducing the size of bounding box used, is proposed in [44] to achieve both high sampling efficiency and high localization accuracy, which most SMC schemes suffer from. Moreover, WMCL can achieve high localization accuracy no matter static networks or mobile networks, especially for relatively high mobile velocity. COMCL (Constraint rules Optimized Monte Carlo Localization Scheme) is proposed in [45] to improve accuracy or reduce bounding box further. A set of rigorous constraint conditions is generated after the upper and lower bounds of optimized constraint rules have been solved for each neighbor seed of the node. And then, a bounding box could be built as sampling area according to these constraint conditions. Constraint conditions can also be used in filtering phase for the proposed scheme so that COMCL adapts more strict filtering conditions than other previous SMC-based localization schemes.

All MCL-based localization schemes, there are two main inferiors generally. The first is that sampling and filtering iteratively consume lots of energy. The other is that higher localization accuracy is achieved through increasing the number of anchors. These bring out extraordinary consequences, especially for WSNs, energy-constraint and economic-constraint applications. Thus, there are some improved spaces for MCL-based localization for mobile WSNs. Besides MCL methods used for mobile WSNs, there are several other localization methods for mobile WSNs, such as localization scheme based on convex method, localization based on geometric constraints, and localization based on perpendicular bisector of a chord [46-48].
In this paper, an improved cross-layer DV-hop localization scheme IMDV-hop is proposed for real-time large-scale aquaculture monitoring and localization system, accompanying with HTC scheme [49] in mobile WSN. At first, inferiorities of original DV-hop scheme are denoted elaborately after brought briefly about it. Then, an improved localization scheme embedded in WLS is proposed based on mitigating these inferiorities from four aspects. Firstly, HTC scheme is combined into IMDV-hop scheme. Secondly, hop count hop $p_{i}$ decreases sharply for adopting HTC algorithm, consequently increases accurate of average distance per hop $d p h_{i}$. Thirdly, distances between known node and anchors adopting $d p h_{i}$ of each anchor, rather than adopting $d p h_{\text {near }}$ which refer to average distance per hop of the nearest anchor. Finally, two critical parameters, correction coefficient $k_{c}$ and weighted coefficient $w_{N_{x}, i}$, are introduced into IMDV-hop scheme to improve localization performance. And then, localization behaviors such as error, delay, and energy consumption are validated adopting NS-2 simulations, taking parameters describing the network into account, such as ratio of anchors $(p)$, node density $(\lambda)$, velocity of nodes $(v)$, localization window $(L W)$, and transmission range $(R)$. Moreover, comprehensive performance comparisons between IMDV-hop and other DV-hop-based schemes and performance comparisons between IMDV-hop and MCL-based schemes are proposed.

The main contributions in this paper are threefold. Firstly, cross layer time-critical localization scheme which combines HTC scheme with IMDV-hop localization scheme embedded a modification of WLS method is proposed. IMDV-hop localization scheme attenuates inferiors of original DV-hop scheme from four aspects. Secondly, comprehensive simulations are presented to validate performance evaluations taking account of $p, \lambda, v, L W, R$. Finally, comparisons between IMDV-hop scheme and other DV-hop-based schemes or MCL-based mobile localization schemes are proposed to validate delay superiority of this time-critical scheme IMDV-hop for static or mobility monitoring networks.

\section{Methods/experimental}

Firstly, we briefly explain the original DV-hop scheme as well as some pending improvements in this scheme. Then, we present our improved localization scheme IMDV-hop-based DV-hop algorithm, accompanying with HTC algorithm for mobile WSNs. The aim of IMDV-hop is to improve the delay behavior for large-scale mobile localization networks, without reducing localization accuracy and energy-saving.

\subsection{The original DV-hop algorithm}

DV-hop localization algorithm, a range-free-based localization scheme, was firstly proposed by 
Niculescu $[25,26]$. It is a suitable solution for normal nodes having three or less neighbor anchors. There are two types of nodes in DV-hop scheme, and one type is anchor nodes equipped with GPS which can obtain their location information. The other is normal nodes pending to be localized. As shown in Fig. 1, although the pending localized node $N_{x}$ has no direct neighbor or reachable anchor, $N_{x}$ can use DV-hop algorithm to be localized. DV-hop scheme consists of following three steps:

Step 1: First, each anchor $A_{i}$ broadcasts a message throughout network containing position of $A_{i}$ and a hop count field hop $p_{i}$ set to 0 . Value of hop increases with the hop during the message broadcasting, which means, hop count value $h o p_{i}$ in the message will be incremented as soon as a node receives this message. Every node $N$ (either anchor or normal node) records position of $A_{i}$ and initializes value of $h_{o p}$ as hop count value in the message. And $h o p_{i}$ is the minimum hop count between $N$ and $A_{i}$. If the same message is received again, node $N$ maintains $h o p_{i}$, and if this received message contains a lower hop count value than $h o p_{i}, N$ will update $h o p_{i}$ with that lower value and relay the message. Otherwise, $N$ will ignore the message. Through this mechanism, each node can obtain the minimum hop count hop $p_{i}$ between each anchor and it separately.

Step 2: Second, when an anchor $A_{i}$ receives positions of other anchors as well as the minimum hop counts to other anchors, $A_{i}$ can calculate its average distance per hop, which is denoted as $d p h_{i} \cdot d p h_{i}$ is calculated as Eq. 1.

$$
d p h_{i}=\frac{\sum_{j=1, j \neq i}^{M} \sqrt{\left(x_{i}-x_{j}\right)^{2}+\left(\mathrm{y}_{i}-\mathrm{y}_{j}\right)^{2}}}{\sum_{j=1, j \neq i}^{M} h_{0} p_{i, j}}
$$

In Eq. $1, M$ is the number of anchors in the network, and node $j$ refers to other anchor different from anchor $i$. hop $i, j$ is the minimum distance between anchor $i$ and anchor $j$ measured by hops. $\left(x_{i}, y_{i}\right)$ and $\left(x_{j}, y_{j}\right)$ refer to coordinates of anchor $i$ and anchor $j$, respectively. Once $d p h_{i}$ is calculated, it will be broadcasted by $A_{i}$. And then, all anchors can obtain all other anchors' $d p h_{i}$, but unknown nodes $N_{x}$ can only maintain value of $d p h_{i}$ broadcasted by the nearest anchor $A_{\text {near }}$ (either one-hop or higher hop neighbor anchor).

When receiving $d p h_{i}$ of $A_{\text {near }} N_{x}$ can obtain distances to each anchor $A_{i}$ (including $A_{\text {near }}$ ), which multiplies $h p_{i, N_{x}}$ (its distance to $A_{i}$ by hop count) by $d p h_{\text {near }}$ (the average distance one hop $d p h_{i}$ for $A_{\text {near }}$ ). This distance is denoted as $d_{i, N_{x}}$. Then, $M$ distances are obtained by node $N_{x}$, which refers to $d_{1, N_{x}}, d_{2, N_{x}}, d_{i, N_{x}}$, till $d_{M, N_{x}}$.

$$
d_{i, N_{x}}=d p h_{\text {near }} \times \text { hop }_{i, N_{x}}
$$

Note that all multiple factor $d p h_{\text {near }}$ is the same value in Eq. 2, which is the average distance one hop for $A_{\text {near }}$ from $N_{x}$.

For example, anchor $A_{1}$ is $40 \mathrm{~m}$ and two hops away from anchor $A_{2}, 100 \mathrm{~m}$ and six hops away from anchor $A_{3}$, and $40 \mathrm{~m}$ and three hops away from anchor $A_{4}$ in Fig. 1. $A_{1}$ can calculate $d p h_{1}$ using Eq. 1 . Hence, $d p h_{1}$ is equal to $(40+100+40) /(2+6+3)=16.36 \mathrm{~m}$. In the similar way,

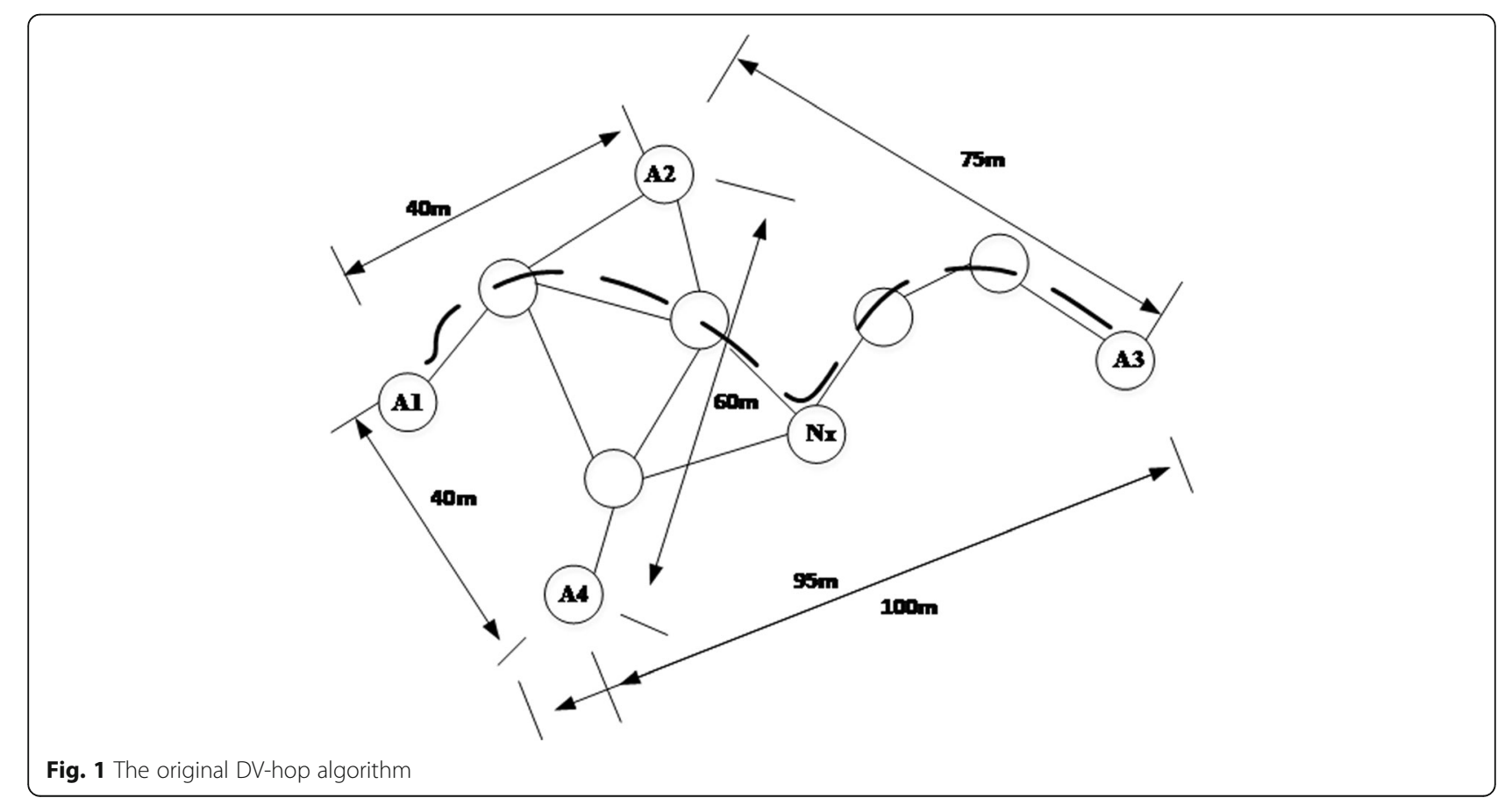


$A_{2}, A_{3}$, and $A_{4}$ calculate the value of $d p h_{2}, d p h_{3}$, and $d p h_{4}$, as $(40+75+60) /(2+5+3)=17.5 \mathrm{~m},(100+75+95) /(6+$ $5+5)=16.88 \mathrm{~m}$, and $(40+60+95) /(3+3+5)=17.73 \mathrm{~m}$, respectively. Then, each anchor $A_{i}(\mathrm{i}=1,2,3,4)$ broadcasts its $d p h_{i}$ in the network, so other anchors and normal sensors receive it. Unknown node $N_{x}$ will maintain the value of $d p h_{2}$, for $A_{2}$ is the nearest anchor to it, and calculate the distance away from $A_{1}, A_{2}, A_{3}$, and $A_{4}$, as $17.5 \times 3=$ $52.5 \mathrm{~m}, 17.5 \times 2=35 \mathrm{~m}, 17.5 \times 3=52.5 \mathrm{~m}$, and $17.5 \times 2=$ $35 \mathrm{~m}$, respectively.

Step 3: Third, when receiving the distance of $N_{x}$ and $A_{i}$, unknown node $N_{x}$ can calculate its estimated position by trilateration or other arithmetic methods as follows: $\left(x_{i}, y_{i}\right)$ in Eqs. 3 and 4 is the coordinate of anchor $A_{i}$.

$$
\begin{aligned}
& \left\{\begin{array}{l}
\left(x_{1}-x\right)^{2}+\left(y_{1}-y\right)^{2}=d_{1}^{2} \\
\ldots \\
\left(x_{M}-x\right)^{2}+\left(y_{M}-y\right)^{2}=d_{M}^{2}
\end{array}\right. \\
& A=\left[\begin{array}{ll}
2\left(x_{1}-x_{M}\right) & 2\left(y_{1}-y_{M}\right) \\
\ldots \\
2\left(x_{M-1}-x_{M}\right) & 2\left(y_{M-1}-y_{M}\right)
\end{array}\right], B \\
& =\left[\begin{array}{l}
x_{1}^{2}-x_{M}^{2}+y_{1}^{2}-y_{M}^{2}+d_{M}^{2}-d_{1}^{2} \\
\cdots \\
x_{M-1}^{2}-x_{M}^{2}+y_{M-1}^{2}-y_{M}^{2}+d_{M}^{2}-d_{M-1}^{2}
\end{array}\right], X=\left[\begin{array}{l}
x \\
y
\end{array}\right]
\end{aligned}
$$

Coordinate $(x, y)$ of unknown node $N_{x}$ can be obtained through standard minimum mean variance estimation method as:

$$
X=\left(A^{T} A\right)^{-1} A^{T} B
$$

\subsection{The motivations for the improved algorithms}

DV-hop algorithm, range-free localization scheme as related above, can localize unknown nodes with less than three neighbor anchors and obtain relative satisfied localization errors with less complexity and less additional hardware. But there are some defects for original DV-hop scheme, and localization performance does not come up to expectations.

Firstly, hop count value hop $i$ can be incremented with message broadcasting if its received hop count is less than the former maintained one, which is related in step 1 of the original DV-hop scheme. Hop count is incremented so long as the message is broadcasted once, no matter if the next node is in the transmission range or not, no matter how node density is. This leads to over-estimate hop count value hop $i$ if node density is relatively high, and subsequently, average hop distance $d p h_{i}$ is underestimated adopting Eq. 1. For example, hop count between anchor $A_{1}$ and anchor $A_{3}$ is 6, and geometrical distance between $A_{1}$ and $A_{3}$ is $100 \mathrm{~m}$, while hop count between anchor $A_{4}$ and anchor $A_{3}$ is 5 , and geometrical distance between $A_{4}$ and $A_{3}$ is $95 \mathrm{~m}$. If hop count is increased with broadcasting, real distance between $A_{1}$ and $A_{3}$ is underestimated.

Secondly, it cannot decide to select which anchor to calculate value of $d p h_{\text {near }}$ if there are several anchors with the same distance away from $N_{x}$. In step 2 of the original DV-hop scheme, node $N_{x}$ maintains $d p h_{i}$, which is the nearest anchor $A_{\text {near }}$ to it, and calculates distances away from all anchors with this value of $d p h_{i}\left(d p h_{\text {near }}\right)$. That is to say, $d p h_{\text {near }}$ is an important factor in localization performance. In Eq. 2, distances of $N_{x}$ away from other anchors can be obtained through hop distance multiplying by $d p h_{\text {nean }}$ and all anchors adopt the same value of $d p h_{i}$ no matter which distance away from this normal $N_{x}$. However, this uniform $d p h_{i}$ can bring out a plenty of localization errors. For example, $N_{x}$ has two two-hop neighbor anchors $A_{2}$ and $A_{4}$ in Fig. 1, and it cannot decide which one it maintains $d p h_{2}$ or $d p h_{4}$. If $N_{x}$ maintains $d p h_{2}$, distance of $N_{x}$ away from $A_{1}, A_{2}, A_{3}$, and $A_{4}$ is 17.5 multiplied by $3,2,3$, and 2 , respectively. While $N_{x}$ maintains $d p h_{4}$, distance of $N_{x}$ away from $A_{1}$, $A_{2}, A_{3}$, and $A_{4}$ is the value of 17.73 multiplied by $3,2,3$ and 2, respectively. Thus, distance $d_{2, N_{x}}$ is relatively accurate, and other distances are relatively inaccurate.

Thirdly, network connectivity also plays an important role in DV-hop localization scheme. Distance between two nodes (either anchor or normal node) is represented by hops, rather than by geometrical distance. Network connectivity exerts a tremendous influence on hop distance, which means hop distance can bring about greater inaccuracy if network connectivity is relatively low. For example, hop distance between anchor $A_{4}$ and anchor $A_{1}$ is three hops through broadcasting in Fig. 1, but geometrical distance is only $40 \mathrm{~m}$ in fact, almost two hops away from each other.

Finally, unknown node can obtain its location through the method of trilateration, which need three known anchors at least, no matter where anchors located. Hops can be enlarged if anchor density is relatively low, which brings about value of $d p h_{\text {near }}$ lower than factual one. And also, unknown node tries to seek farther anchors through two or more hop relay transmission, which can bring about a plenty of unnecessary energy consumption.

These inconveniences can be overcome through several modified method. Firstly, hop counts between nodes, either between an anchor and unknown node or between an anchor and another anchor, are decreased through adopting HTC scheme. Secondly, localization messages can be exchanged in one-hop or two-hop transmissions, which decreases energy consumed in plenty of broadcast. Then, network connectivity can be improved through increasing node density. And also, model's accuracy is improved largely through involving in two parameters $k_{c}$ and $w_{N_{x}, i}$. Lastly, $d_{i, N_{x}}=d p h_{i}$ $\times h o p_{i, N_{x}}$ instead of $d_{i, N_{x}}=d p h_{\text {near }} \times h o p_{i, N_{x}}$ is used to 
calculate the distance of $N_{x}$ and $A_{i}$, accompanying with WLS method.

\subsection{Method}

Several assumptions are presented before deriving IMDV-hop models:

1. Nodes, normal nodes ( $N_{x}$ denoted as in IMDV-hop) or anchors ( $A$ denoted as in IMDV-hop), are randomly located in circle plane, according to a twodimensional Poisson distribution with a density of $\lambda$. Parameters $\lambda$ and $p$ denote the density of Poisson distribution for nodes including anchors and unknown nodes and the ratio of anchors, respectively. We assume identical range $R$ of communication, interference, and carrier sensing. That is, the number of anchors can be denoted as $N_{\text {anchor }}(h o p=1)=\lambda p \pi R^{2}$ in one-hop transmission range, and the number of normal nodes can be denoted as $N_{x}(h o p=1)=\lambda(1-p) \pi R^{2}$.

2. Time is divided into discrete intervals, and a sensor node localizes itself in each localization window $L W$, which is constituted by several intervals. Environment parameters can be collected and transmitted periodically, that is, at the beginning of each $L W$. Nodes can also execute localization scheme IMDV-hop at the beginning slot of each $L W$. And so, we can simplify mobile localization as relatively static localization process in each $L W$, and mobile patterns in each $L W$ can be ignored, which are related elaborately in [37].

3. In our monitoring aquaculture network, we pay close attention to environmental information such as culture temperature, $\mathrm{PH}$ value, or environment oxygen content and exact locations which these information are brought about. Antennas of nodes are floating upon the water, and information collecting parts of them are soaking under the water, which can be presented in Fig. 3. Thus, all signal transmissions happen upon the water, without taking signal attenuation into account.

4. Nodes including anchors regardless of locations are bestowed on the fair chance to transmit messages with CSMA/CA scheme to its neighbors through one-hop or two-hop transmission heterogeneously and nonpreemptively, as similar to the HTC algorithm [49].

And now, IMDV-hop scheme accompanying with HTC algorithm is presented elaborately.

\subsubsection{Step 1: localization request}

First, $L W$ and $h o p_{i}$ are initialized, which are set to $L W_{0}$ and 0 , respectively. After network initialization completing, each node (either anchor or unknown node) can be aware which hop belongs to this time slot (that is, in this
$L W$ period). Also, all nodes can establish their one-hop neighbor list and update it every slot of $L W$. As localization request phase starts up, an unknown node $N_{x}$ in its one-hop circle ( $d<R$, which can be seen from Fig. 2) can confirm its one-hop anchor neighbors $A_{1 i}$ (anchors in one-hop) in its transmission range just in one-hop circle. And then, node $N_{x}$ will send a HTC frame to anchors $A_{1 i}$, and HTC frame contains the value of $L W$ and all one-hop neighbors including one-hop anchor neighbors $A_{1 i}$ of $N_{x}$. If the number of $A_{1 i}$ is greater than or equal to 3 , node $N_{x}$ can directly go to execute DV-hop scheme as original DV-hop scheme. If the number of $A_{1 i}$ is less than 3 , it needs to spread its HTC frame to two-hop neighbors. And also, two-hop neighbors including anchor neighbors $A_{2 i}$ (anchors in two-hop of $N_{x}$ ) are added to this frame. If the sum of $A_{1 i}$ and $A_{2 i}$ is greater than or equal to 3, localization scheme can be promoted. Otherwise, this localization can be a failure.

It is to be noted that we execute IMDV-hop scheme in two-hop networks (either two-hop of $N_{x}$ or two-hop of anchors) for several reasons. Firstly, HTC algorithm can be presented the most of the advantage in two-hop networks for avoiding transmission collisions. Secondly, node density of this work is relatively high, which can satisfy the condition of the number of $A_{i}$ is greater than or equal to 3 in two-hop circle. The last and foremost reason is that node $N_{x}$ or anchor $A_{i}$ need not to establish or maintain routings to transmit information of hop count, hop distance, or environmental parameters, which can save overwhelming majority of energy in WSNs. After this request phase accomplishes, unknown node $N_{x}$ can obtain its one-hop and two-hop neighbors including anchor neighbors, and its one-hop and two-hop neighbor anchors can obtain its HTC frame.

\subsubsection{Step 2: hop information exchange}

Unlike broadcasting HopCount message to obtain the minimum hop count as original DV-hop scheme, IMDV-hop fusing HTC algorithm can obtain hop count as follows: After localization request phase finishes, one-hop anchor neighbor $A_{1 i}$ broadcasts a HTC frame containing location of $A_{1 i}$, ID of $A_{1 i}$, circumstance parameters such as culture temperature, $\mathrm{PH}$ value, and environment oxygen content, and also a hop count field $h o p_{i}$. There are three cases for obtaining hop ${ }_{i}$. The first one is that the number of anchor nodes in the intersection area for one-hop neighbors of $A_{1 i}$ and one-hop neighbors of $N_{x}$ is greater than or equal to 3 , each minimum hop count $h o p_{i}$ is set to 1 , and the $\operatorname{sum} \sum h o p_{i j}(\mathrm{i} \neq \mathrm{j})$ is set to the number of other anchors (case 1). If the number of anchors in the intersection area for one-hop neighbors of $A_{1 i}$ and one-hop neighbors of $N_{x}$ is less than 3 , hop count hop is set to 1 if other anchors in 


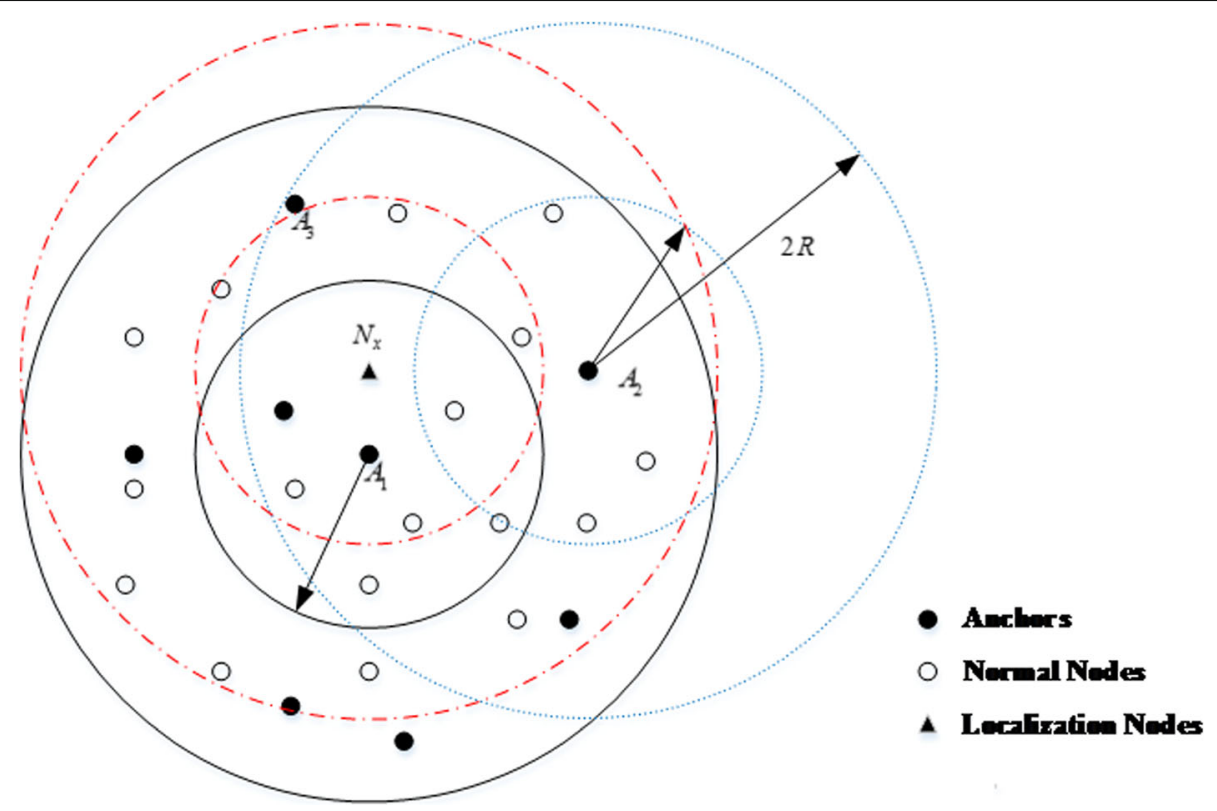

Fig. 2 The establishment of IMDV-hop scheme. $N_{x}$ is the unknown node and $A_{i}$ is the anchors

one-hop of $A_{1 i}$, and set to 2 if other anchors in two-hop of $A_{1 i}$ (anchors in one-hop). Sum $\sum h o p_{i j}(\mathrm{i} \neq \mathrm{j})$ is set to $\left(N_{A_{1 i}}+2 N_{A_{2 i}}-1\right)$ (case 2). Of course, if number of anchors in one-hop of $N_{x}$ is less than 3, IMDV-hop can calculate the location of $N_{x}$ by expanding to anchors $A_{2 i}$ in two-hop of $N_{x}$, and $\sum h o p_{i j}(\mathrm{i} \neq \mathrm{j})$ is the same as the second one as related above (case 3). And also, each anchor involved in localization calculation can be a router to transmit monitoring information, which performs real-time environmental monitoring in our aquaculture sensor networks.

$$
\begin{gathered}
\sum h o p_{i j}(\mathrm{i} \neq \mathrm{j})= \begin{cases}N_{A_{1 i}}-1 & \text { case } 1 \\
N_{A_{1 i}}+2 N_{A_{2 i}}-1 & \text { case } 2,3\end{cases} \\
\left\{\begin{array}{l}
N_{A_{1 i}}=4 p \lambda \int_{d / 2}^{2 R} \sqrt{4 R^{2}-x^{2}} d x \\
N_{A_{2 i}}=p \lambda \pi R^{2}-4 p \lambda \int_{d / 2}^{2 R} \sqrt{4 R^{2}-x^{2}} d x- \\
\left.2 p \lambda\left(\int_{\left(3 R^{2}-d^{2}\right) / 2 d}^{R} \sqrt{R^{2}-x^{2}} d x-\int_{d+\left(3 R^{2}-d^{2}\right) / 2 d}^{2 R} \sqrt{4 R^{2}-x^{2}} d x\right)\right)
\end{array}\right.
\end{gathered}
$$

\subsubsection{Step 3: distance calculations}

Like the calculation of distances between anchors and pending localized nodes, anchors in IMDV-hop fusing HTC algorithm can obtain distances as follows: After $A_{1 i}$ or $A_{2 i}$ receive positions of other anchors as well as the minimum hop count hop ${ }_{i}$, anchor $A_{1 i}$ or $A_{2 i}$ can calculate its average distance per hop, which is denoted as $d p h_{i}$. In Eq. 6, $\left(x_{i}, y_{i}\right)$ and $\left(x_{j}, y_{j}\right)$ refers to coordinates of anchor $i$ and anchor $j$, respectively. Once $d p h_{i}$ is calculated, it will be broadcasted by $A_{1 i}$ or $A_{2 i}$, and then, all anchor nodes can obtain all other anchors' $d p h_{i}$ as Eq. 6.

$$
d p h_{i}=\frac{\sum_{i \neq j} \sqrt{\left(x_{i}-x_{j}\right)^{2}+\left(\mathrm{y}_{i}-\mathrm{y}_{j}\right)^{2}}}{\sum_{i \neq j} h o p_{i j}}
$$

Unknown node $N_{x}$ can maintain $d p h_{i}$ broadcasted by all anchors $A_{1 i}$ or $A_{2 i}$, not similar to the value of $d p h_{i}$ in original DV-hop for node $N_{x}$. When receiving $d p h_{i}$, unknown node $N_{x}$ can calculate the distance to each anchor $A_{1 i}$ or $A_{2 i}$, which is denoted as $d p h_{N_{x}, i}$. Distance $d p h_{N_{x}, i}$ is the value of $d p h_{1 i}$ if anchor $A_{1 i}$ is in one-hop of $N_{x}$, and $2^{*} d p h_{2 i}$ if anchor $A_{2 i}$ is in two-hop of $N_{x}$. That is to say, value $d p h_{N_{x}, i}$ is the multiplication of $d p h_{i}$ (distance per hop for anchor $A_{i}$ ) and parameter 1 or 2 (its distance to $A_{i}$ by hop count), which is not similar to the original DV-hop.

$$
d p h_{N_{x}, i}= \begin{cases}d p h_{1 i} & \text { case } 1 \\ 2^{*} d p h_{2 i} & \text { case } 2,3\end{cases}
$$

\subsubsection{Step 4: location calculations}

In this algorithm, we can introduce a correction coefficient $k_{c}$ to improve localization accuracy of distances 
between unknown node $N_{x}$ and anchors, which is shown in Eq. 8.

$$
k_{c}=\left.\frac{d p h_{e s t}^{i, j}-d p h_{\text {true }}^{i, j}}{d p h_{\text {true }}^{i, j}}\right|_{i \neq j}
$$

In Eq. 8, actual distance $d p h_{\text {true }}^{i, j}$ of each anchor can be calculated using geometric method involving actual coordinates, $d p h_{\text {true }}^{i, j}=\sqrt{\left(x_{i}-x_{j}\right)^{2}+\left(y_{i}-y_{j}\right)^{2}}$ and estimation distance $d p h_{e s t}^{i, j}$ is calculated through Eq. 1 or Eq. 6. In the same way, distance of $N_{x}$ and anchors can be calculated using Eq. 9. Actual distance $d p h_{t r u e}^{N_{x}, i}$ and estimation distance $d p h_{e s t}^{N_{x}, i}$ of $N_{x}$ and anchors can be calculated as related above. We can consider the difference between $d p h_{\text {true }}^{N_{x}, i}$ and $d p h_{e s t}^{N_{x}, i}$ as the similar to difference between $d p h_{\text {true }}^{i, j}$ and $d p h_{\text {est }}^{i, j}$ for our uniform randomly networks.

$$
d p h_{\text {true }}^{N_{x}, i}=\frac{d p h_{e s t}^{N_{x}, i}}{1+k_{c}}
$$

If the coordinate of unknown node $N_{x}$ is denoted as $(x, y)$, the location of $N_{x}$ is then calculated by using following system of Eqs. 10 and 11, in which $d p h_{\text {true }}^{N_{x}, i}$ can be presented as a simple form $d_{i}$. And $n$ is the number of anchors, that is, the simple form for $N_{A_{i}}$.

$$
\begin{aligned}
& \left.\begin{array}{l}
\sqrt{\left(x-x_{1}\right)^{2}+\left(y-y_{1}\right)^{2}}=d_{1} \\
\sqrt{\left(x-x_{2}\right)^{2}+\left(y-y_{2}\right)^{2}}=d_{2} \\
\cdots \\
\sqrt{\left(x-x_{n}\right)^{2}+\left(y-y_{n}\right)^{2}}=d_{n}
\end{array}\right\} \\
& \sqrt{\left(x-x_{1}\right)^{2}+\left(y-y_{1}\right)^{2}}-\sqrt{\left(x-x_{n}\right)^{2}+\left(y-y_{n}\right)^{2}}=d_{1}-d_{n} \\
& \sqrt{\left(x-x_{2}\right)^{2}+\left(y-y_{2}\right)^{2}}-\sqrt{\left(x-x_{n}\right)^{2}+\left(y-y_{n}\right)^{2}}=d_{2}-d_{n} \cdots \\
& \left.\sqrt{\left(x-x_{n-1}\right)^{2}+\left(y-y_{n-1}\right)^{2}}-\sqrt{\left(x-x_{n}\right)^{2}+\left(y-y_{n}\right)^{2}}=d_{n-1}-d_{n}\right\}
\end{aligned}
$$

Squaring both sides and simplifying Eq. 11, we can obtain Eq. 12 as the same as [30].

$$
\left.\begin{array}{l}
-2 x\left(x_{1}+x_{n}\right)-2 y\left(y_{1}+y_{n}\right)+2\left(x^{2}+y^{2}\right)=d_{1}{ }^{2}+d_{n}{ }^{2}-\left(x_{1}^{2}+y_{1}^{2}+x_{n}^{2}+y_{n}^{2}\right) \\
-2 x\left(x_{2}+x_{n}\right)-2 y\left(y_{2}+y_{n}\right)+2\left(x^{2}+y^{2}\right)=d_{2}{ }^{2}+d_{n}{ }^{2}-\left(x_{2}^{2}+y_{2}^{2}+x_{n}^{2}+y_{n}^{2}\right) \ldots \\
-2 x\left(x_{n-1}+x_{n}\right)-2 y\left(y_{n-1}+y_{n}\right)+2\left(x^{2}+y^{2}\right)=d_{n-1}{ }^{2}+d_{n}{ }^{2}-\left(x_{n-1}^{2}+y_{n-1}^{2}+x_{n}^{2}+y_{n}^{2}\right)
\end{array}\right\}
$$

$Q=\left[\begin{array}{llc}-2 x\left(x_{1}+x_{n}\right) & -2 y\left(y_{1}+y_{n}\right) & 1 \\ -2 x\left(x_{2}+x_{n}\right) & -2 y\left(y_{2}+y_{n}\right) & 1 \\ \cdots & & \\ -2 x\left(x_{n-1}+x_{n}\right) & -2 y\left(y_{n-1}+y_{n}\right) & 1\end{array}\right]$

$H=\left[\begin{array}{l}d_{1}{ }^{2}+d_{n}{ }^{2}-\left(x_{1}^{2}+y_{1}^{2}+x_{n}^{2}+y_{n}^{2}\right) \\ d_{2}{ }^{2}+d_{n}{ }^{2}-\left(x_{2}^{2}+y_{2}^{2}+x_{n}^{2}+y_{n}^{2}\right) \\ \cdots \\ d_{n-1}{ }^{2}+d_{n}{ }^{2}-\left(x_{n-1}^{2}+y_{n-1}^{2}+x_{n}^{2}+y_{n}^{2}\right)\end{array}\right], Z=\left[\begin{array}{l}x \\ y \\ k\end{array}\right]$

$$
Q Z=H
$$

WLS algorithm is adopted to solve the coordinate of $N_{x}$ in our IMDV-hop scheme, to improve location accuracy. In WLS method, unknown parameters in Eq. 14 can be presented as:

$$
Z=\left(Q^{\prime} W^{\prime} W Q\right)^{-1} Q^{\prime} W^{\prime} W H
$$

In which, $W$ is the weighted matrix which presents the influence of distance between anchors and unknown node $N_{x}$, transmission range, and distances between an anchor and other anchors. For example, an anchor is farther away from unknown node $N_{x}$, greater error is brought in localization for $N_{x}$. Thus, the weight of this anchor can be set to a lower value. And also, transmission range and distance between an anchor and other anchors play an important role in localization error.

$$
W=\left[\begin{array}{llll}
w_{N_{x}, 1} & 0 & \ldots & 0 \\
0 & w_{N_{x}, 2} & \ldots & 0 \\
\ldots & & & \\
0 & 0 & \ldots & w_{N_{x}, n-1}
\end{array}\right]
$$

Weight $w_{N_{x}, i}$ in Eq. 15 is taken as the inverse of the minimum number of hops between each anchor and $N_{x}$ in $[31,50]$. Not only the minimum number of hops as $[31,50]$, the weight $w_{N_{x}, i}$ should also take transmission range $R$ and $h o p_{i j}$ into account in this work, which is demonstrated as Eq. 16.

$$
w_{N_{x}, i}=\frac{1}{h o p_{N_{x}, i}} \frac{1}{N_{A_{i}}-1} \sum_{k=1}^{N_{A_{i}}-1}\left(1-\frac{h_{o p_{i k}}^{2}}{R^{2}}\right)
$$

In Eq. 16, the number of anchors can be presented as $N_{A_{i}}$ as shown in Eq. 5. hop ${ }_{N_{x}, i}$ and hop $p_{i k}$ denote distance between $N_{x}$ and anchor $i$ and distance between anchor $i$ and other anchor $k$, respectively. Weight values influenced by these three parameters play important roles on localization accuracy, which can be analyzed through 
simulations in Section 4. It is to be noted that hop $p_{i k}$ can be denoted as 1 if anchork in one-hop of anchor $i$ or 2 if anchor $k$ in two-hop of anchor $i$, respectively.

IMDV-hop scheme can be illustrated as flow chart in Fig. 3. When an unknown node $N_{x}$ is being in the state of pending localization, it will execute four steps complying with the step of Fig. 3. In IMDV-hop scheme, $N_{x}$ initializes and maintains $L W$ and HTC table, and anchors initialize and maintain their coordinates, IDs, hop $p_{i}, L W$ and HTC table. At first, $N_{x}$ initializes $L W=L W_{0}$ and HTC table (steps 1-3). After localization request finishes (step 4), IMDV-hop goes to localization stage. Anchors involving one-hop and two-hop broadcast and exchange their initialization information containing their coordinates, IDs, hop $i$, and HTC table (steps 5-6). After obtaining hop counts, anchors can calculate its distance

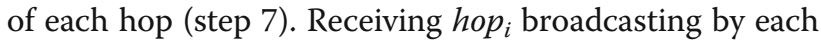
anchor, unknown node $N_{x}$ calculates the distance away from each anchor, which multiples value hop $p_{i}$ of each anchor by the hop away from this anchor, involving $k_{c}$ (step 8). Then, node $N_{x}$ executes WLS method to obtain its location (step 9). If $L W$ is decreasing to 0 , localization of this period fails.

\subsection{Experimental}

As assumptions related in Section 3.3, nodes' structural representation in our aquaculture WSNs are illustrated in Fig. 4a, with antennas fixing upon the float, which floats upon water. Perceptive part is soaking under water, which collects circumstance information. Sensor nodes collect environmental parameters, such as culture temperature, $\mathrm{PH}$ value, or environment oxygen content, and transmit this information to sinks shown in Fig. $4 \mathrm{~b}$. Simultaneously, locations of normal nodes can also be obtained through executing IMDV-hop scheme.
(5)

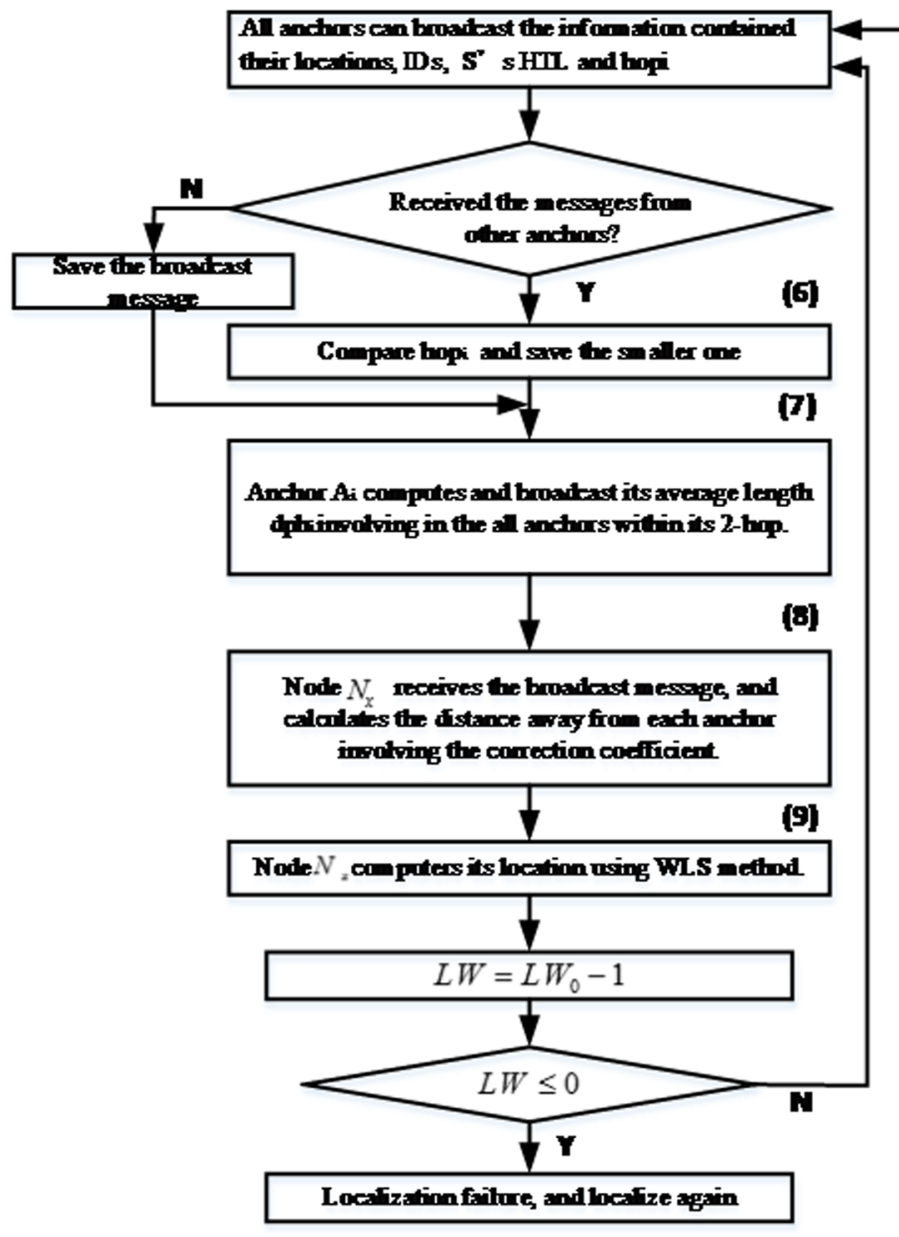

[1]

Node $N_{x}$ senils a Reques flacolizadileasrage which contains the information of $L W$ value andHTC thle
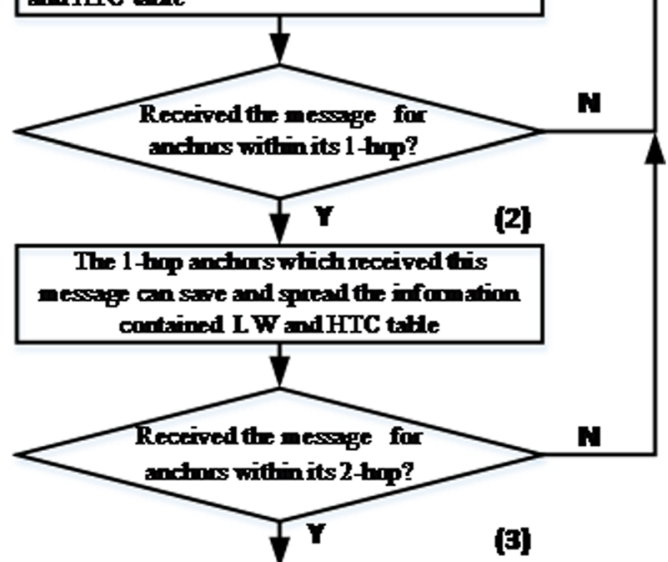

The 2-hop anchos whinreceived this messabe can sore ani spread the information of LW andHTC table

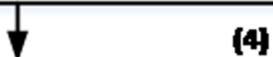

The localization rectesting stage finishes, and the localizatinn stage stants.

Fig. 3 The flow chart of IMDV-hop scheme 
Physical node in this aquaculture network is illustrated in Fig. 5, with the antennas fixing upon the float, and sensors immerge in the water to apperceive and collect circumstance parameters. And sensors including anchors and unknown nodes are floating randomly in aquaculture farm, without taking water currents and waves into account.

We present extensive simulations to validate the accuracy of evaluated metrics for localization error, delay, and energy consumption of IMDV-hop scheme using NS-2 simulator according to previous analyses. NS-2 is a popular discrete-event simulator which was originally designed for wired networks and has been subsequently extended to support wireless simulations. Localization accuracy, delay, and energy consumption are validated through extensive comprehensive simulations which are derived based on analyses of different parameters such as $p, \lambda, v$, and $L W$. And also, comprehensive behavior comparisons between IMDV-hop scheme and DV-hop-based localization schemes are proposed to validate some delay superiority of this time-critical scheme IMDV-hop. And performance comparisons of IMDV-hop with MCL-based localization scheme are also involved in a certain extent, according to velocity of nodes.

Nodes are randomly located in the circle according to a two-dimensional Poisson distribution as Fig. 6, and the case of three-dimensional distribution, such as the localization scheme of $[6,51]$, cannot take account in this paper. Experimental setups of NS-2 simulator used to conduct validations are similar to presentations in [49] in detail, and parameters involved in CSMA/CA scheme, such as UnitSuperframeSlot, BaseSlotDuration, and packet length, are also similar with those of [49], and other specific parameters touched upon simulations, such as $L W$ and $v$, are listed on the head of result figures. It is noted that $L W$ can be scaled by the number of UnitSuperframeSlot. Propagation delay can be ignored in our scheme simulations. We validate performance of our IMDV-hop firstly. Then, we compare performance of our scheme with that of previous schemes such as ADV-hop [31], HDV-hop scheme [33], and Selective-3-anchor scheme [36]. And also, mobility performance of IMDV-hop scheme is compared with that of WMCL scheme [44]. Our simulation results are the mean values derived from 30 experience values for each scenario.

\section{Results and discussion}

\subsection{Localization error validations}

As related in Section 3.3 above, localization error in IMDV-hop is related to correction coefficient $k_{c}$ and weight factor $w_{N_{x}, i}$, which ultimately is related to the minimum hop count, hop size, number of anchors, and $L W$ (in fact, intermittent mobile period). And also, we take $v$ into account for localization error. Symbols of "sim" and "ana" in all figures are denoted as abbreviations of simulation results and analysis results, respectively.

With $p$ increasing, error decreases as shown in Fig. 7a. Either HTC algorithm or IMDV-hop scheme, high anchor ratio means that anchors in one-hop of $N_{x}$ are enough for localizing unknown node, dispensing with spreading HTC table to two-hop neighbors for normal nodes in localization stage and broadcasting hop information to two-hop anchor neighbors for anchors in information exchange stage. And also, with $p$ increasing, weight $w_{N_{x}, i}$ of each anchor decreases, each distance between an anchor and unknown node can only play an insignificant role on localization accuracy. Moreover, with anchor number increasing, accuracy of correction coefficient $k_{c}$ increases, consequently location for $N_{x}$ has more accuracy shown in Eqs. 8 and 16.

Node density plays the similar role on localization behaviors as that of anchor ratio shown as Fig. 7b. As $L W$ is increasing, the error decreases. If each
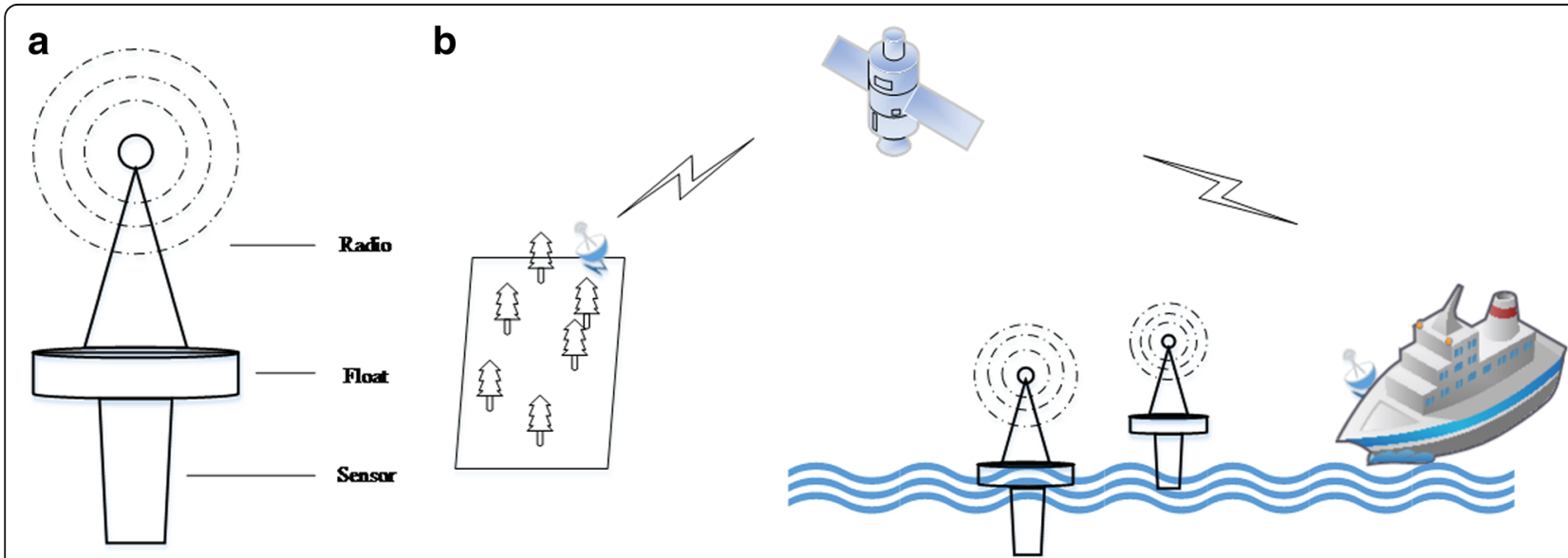

Fig. 4 a The structural representation of nodes in this aquaculture network. $\mathbf{b}$ The structural representation of monitoring and localization system in this aquaculture network 


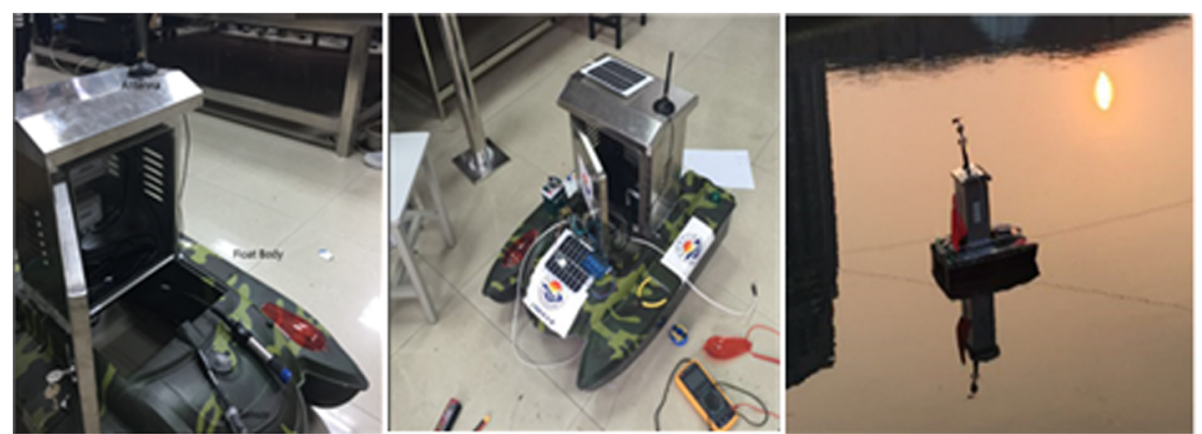

Fig. 5 The physical nodes in this aquaculture network

localization period is not enough to transmit localization information, unknown node or anchors need to retransmit these messages or decide localization failure in this period. And also, nodes maybe are wasted time after localized when $L W$ is too high. For example, error of $L W=40$ is higher than that of $L W=20$ and $L W=10$. With anchor ratio increasing, error for $L W=10$ varies sharply in Fig. 7b.

We only consider low velocity or intermittent mobility for nodes in our aquaculture networks. Velocity is more than 5, error increases sharply, and then stays in a relatively high state shown in Fig. 7c. And error performance presents some independent behaviors with the increasing of node or anchor density, mobility velocity is shown in Fig. $7 \mathrm{a}-\mathrm{c}$. For example, error maintains a stable value when anchor ratio is higher than $20.4 \%$ at $\lambda=0.04$.

\subsection{Delay validations}

Delay is the most important character in our time-critical localization system, and we always attempt to improve behavior of delay in order to obtain real-time monitoring and localizing, ensuring the health of culture objects. As anchor ratio is increasing, localization delay decreases. One-hop anchors in one-hop of $N_{x}$ are greater than or equal to $3 ; N_{x}$ needs to transmit its HTC table or $L W$ only once, which saves plenty of time shown in Fig. 8a.

If node density increases, more nodes including anchors will access the channel simultaneously, which leads to retransmission increasing. Consequently, delay behavior will become inferior which is demonstrated in Fig. 8b. And also, relative optimal value of $L W$ is between 10 and 20 , that is, $L W$ is less than 10, unknown node has no enough time to localize itself in one period $L W$. On the other hand, unknown node need plenty of time to wait next

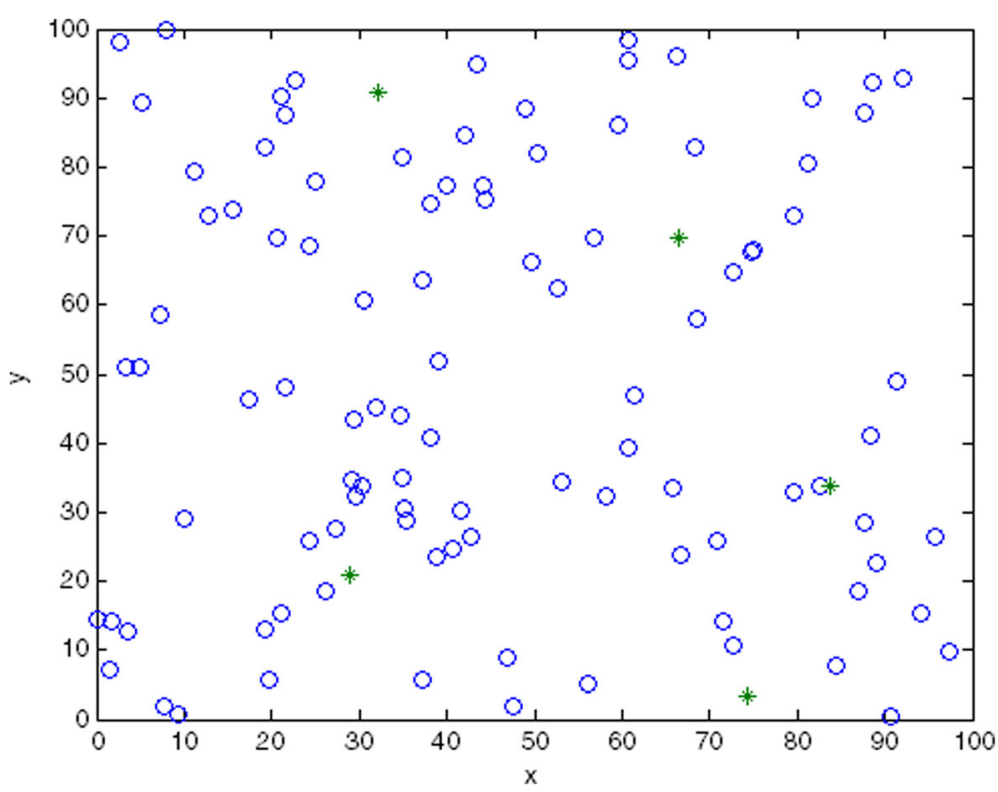

Fig. 6 Example of nodes distribution which contains the unknown nodes (dots) and anchor nodes (stars) 

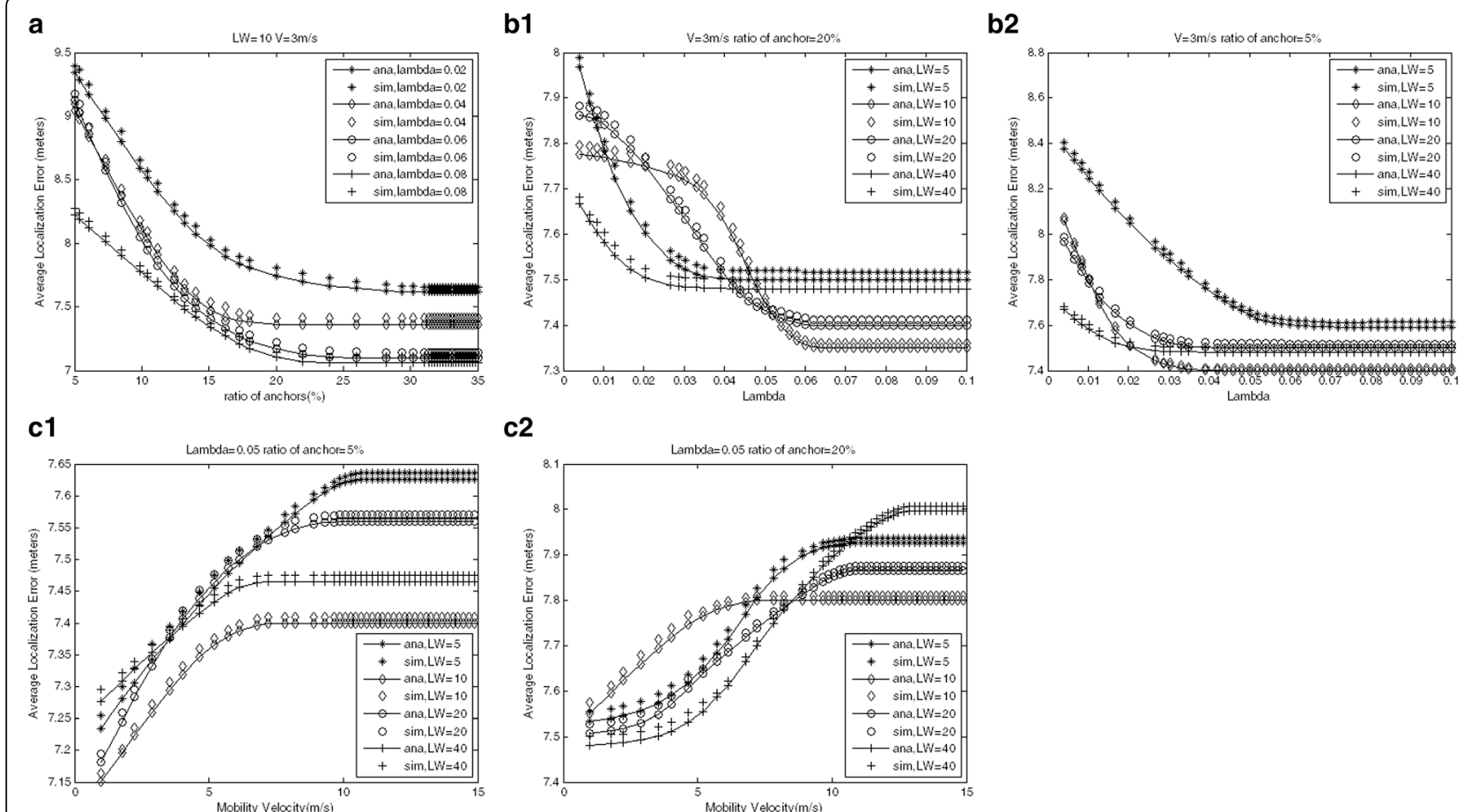

Fig. 7 a Localization error as a function of anchor ratio. b Localization error for localization window as a function of node density (concluding unknown nodes and anchors). c Localization error for localization window as a function of mobility velocity
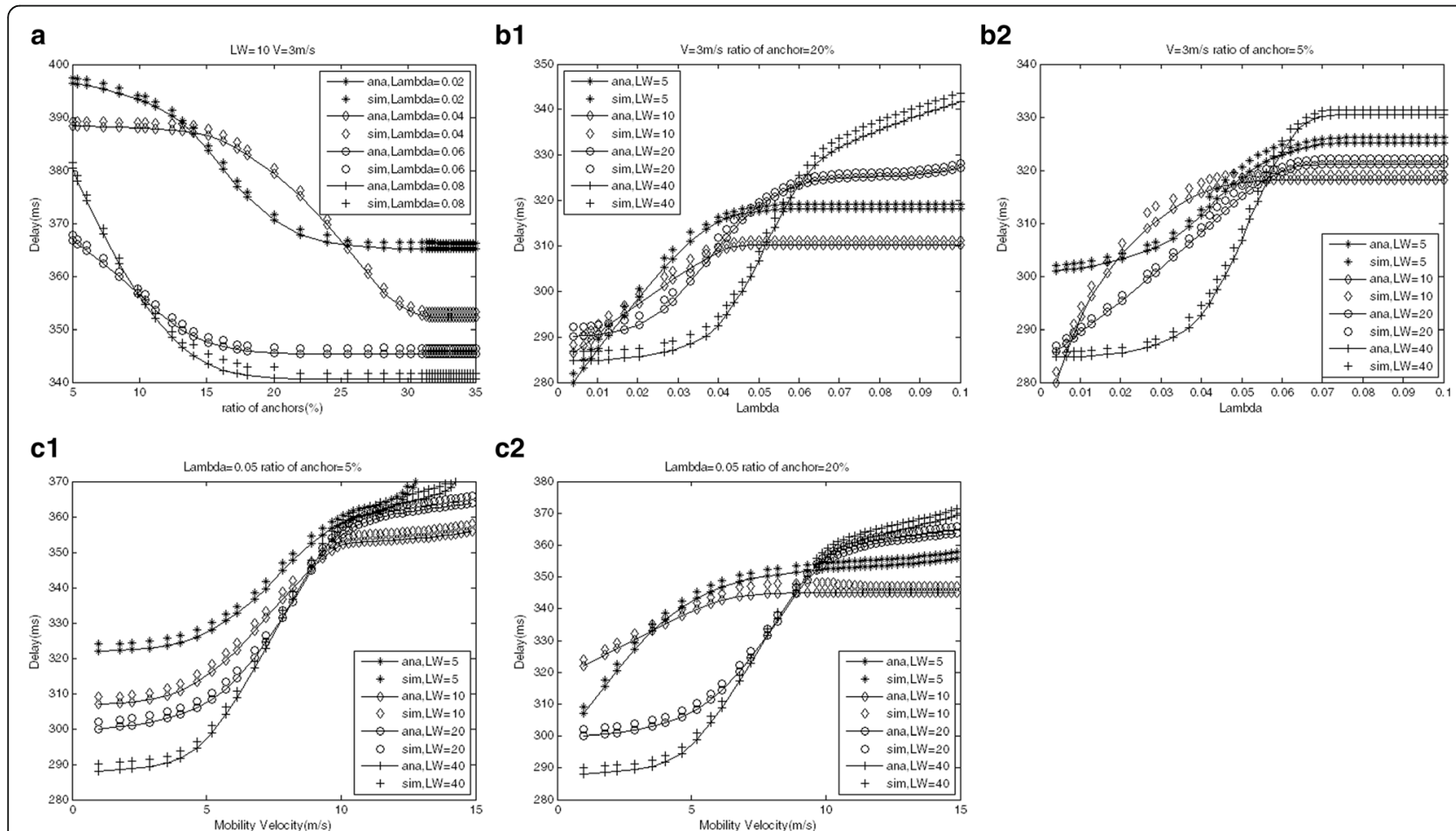

Fig. 8 a Localization delay for node density as a function of anchor ratio. $\mathbf{b}$ Localization delay for localization window as a function of node density. c Localization delay for localization window as a function of mobility velocity 
intermittent mobile period to be localized if $L W$ is too large, such as $L W=40$ in Fig. 8b.

With $v$ increasing, localization delay increases. When $v$ is more than 5, delay increases sharply, especially when anchor ratio is relatively low as shown in Fig. 8c. IMDV-hop scheme presents relatively weak mobility behaviors as shown in Fig. 8c. And, delay largely depend on the value of $v$, especially when $v>5 \mathrm{~m} / \mathrm{s}$.

\subsection{Energy validations}

Energy consumption is an important metric in WSNs, and we also analyze it elaborately. With anchor ratio increasing, localization energy decreases. Shown in Fig. 9a, energy consumption in one $L W$ presents an optimal behavior for $\lambda=0.04$ when anchor ratio is less than $12.56 \%$.

\subsection{Preformation comparisons}

Analysis and simulation results shown above are comprehensive for applications, and we can compare performance metrics of IMDV-hop mechanism with those of other DV-hop schemes. IMDV-hop is used for time-critical monitoring and detection application, in which minimized delay is the most important target. Through comprehensive comparisons, we can derive that delay performance metrics of IMDV-hop scheme accompanying with HTC algorithm are obviously improved over other schemes such as [31, 33, 36], while localization error and energy efficiency are improved over others on the conditions of more node density and less mobility velocity.

ADV-hop in [31] reduces localization errors using WLS method and other improved methods. ADV-hop scheme takes the weight as the inverse of the minimum number of hop count between unknown node and each anchor. In fact, weight factor has also business with anchor number, transmission range and hop counts among anchors. So, ADV-hop can present some inferior performance for some occasions, especially for delay behavior.

Three estimated distance values away from three different anchors are sufficient for unknown node to calculate its location related in selective 3-anchor DV-hop (selective-3-anchor) [36]. Based on the first two steps of original DV-hop, an unknown node can obtain a group of candidates to calculate its location. Then, it chooses the best 3-anchor group to establish estimated position using iterative method. Of course, this best 3-anchor group can also include two-hop neighbor anchors or higher hop anchors to participate in the trilateral method. And also, computational complexity of searching out this best 3 -anchor group using iterative method is $O\left(\mathrm{~m}^{3}\right)$, in which $m$ is the number of anchors. Relatively high computational complexity consumes plenty of energy, which is unsuitable for WSNs and is also incomparable to IMDV-hop.
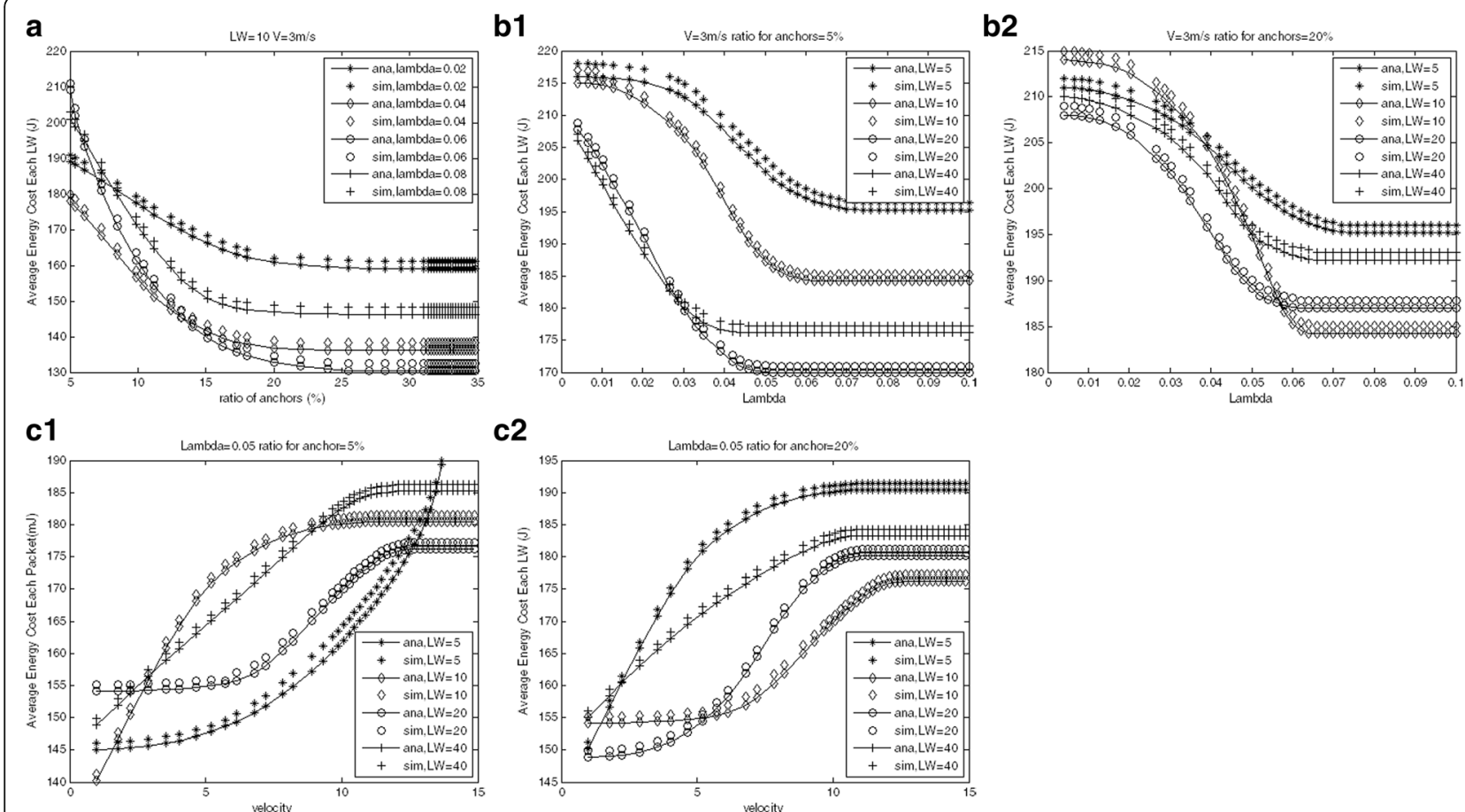

Fig. 9 a Localization energy for node density as a function of anchor ratio. $\mathbf{b}$ Localization energy for localization window as a function of node density. c Localization energy for localization window as a function of mobility velocity 

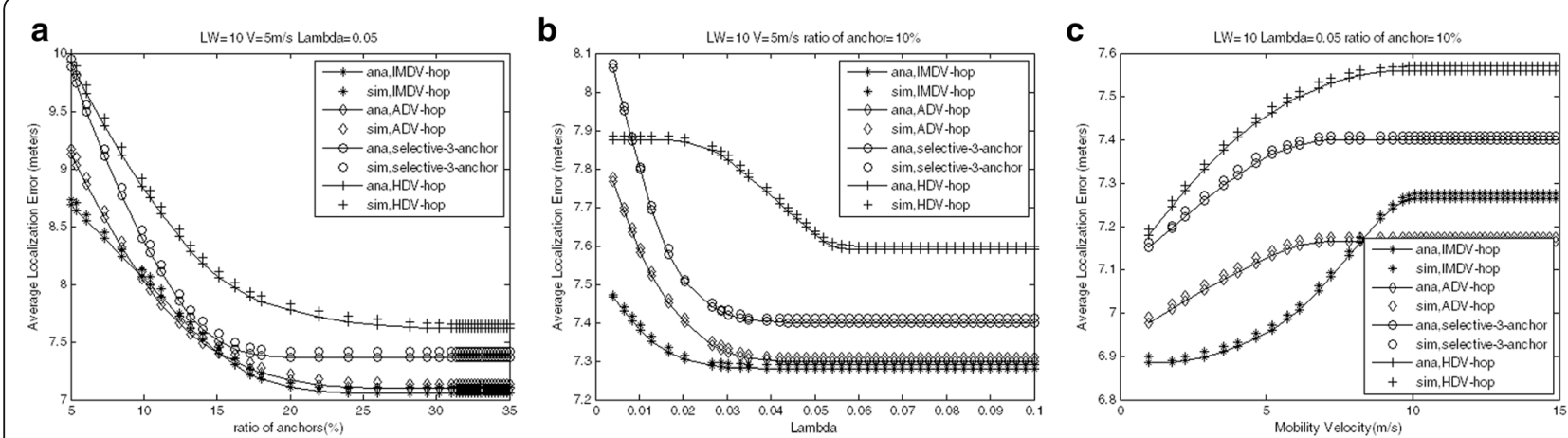

Fig. 10 Localization error comparisons. a Localization error comparisons for different schemes based on the ratio of anchors. $\mathbf{b}$ Localization error comparisons for different schemes based on node density. c Localization error comparisons for different schemes based on mobility velocity

Hybrid DV-hop (HDV-hop) in [33] is suitable for localizing events in hostile environments, in which anchors are deployed on perimeters of networks rather than scattering them inside hostile terrain. Consequently, unknown node which localizes itself will transmit localized messages, including hop count, hop size and the distance of this unknown node and each anchor, transverse two or more hops. This consumes plenty of energy which is intractable to normal monitoring WSNs.

We can set parameters of these three schemes as similar to IMDV-hop scheme in order to compare behaviors between IMDV-hop and other three schemes. For example, in HDV-hop scheme, anchors are deployed on the perimeters of networks, and locations of unknown nodes are similar to those of IMDV-hop. And also, those three localization schemes are designed for static localization, but they also can be adopted for mobile localization, such as mobile scenarios in Section 4.2.4 of [36]. We can set the same mobile environments for all these schemes as IMDV-hop. Localization for all these three schemes and IMDV-hop can executed at the beginning slot of each $L W$. Mobile behaviors are compared for cases of low velocity.
Localization error compares demonstrate that localization error presents the prior behaviors when anchor ratio is higher than 14.65\% and less than 9.45\% shown in Fig. 10a. IMDV-hop shows better localization accuracy with the variety of node density shown in Fig. 10b. Nodes including anchors increasing, unknown node can execute HTC scheme in two-hop sensor networks, which increases the accuracy of hop count, hop size and distance of unknown node and each anchor. IMDV-hop scheme is designed for mobile WSNs on the condition that nodes including anchors move intermittently. We take low mobility velocity into account in IMDV-hop, and localization accuracy shows better behaviors for low velocities shown in Fig. 10c.

We always devote in improving delay behavior for IMDV-hop, a time-critical monitoring and localization scheme for WSNs. Shown in Fig. 11, delay compares demonstrate that delay of it presents prior behaviors when anchor ratio is higher than $7.23 \%$, yet shows less priority for higher mobility velocities such as $v \geq 8.12 \mathrm{~m} / \mathrm{s}$. Energy consumption comparisons are similar to that of delay behavior.

Nearly all of DV-hop-based schemes are designed for localizing static WSNs, but these schemes can also be executed for localizing mobility WSNs with relatively low
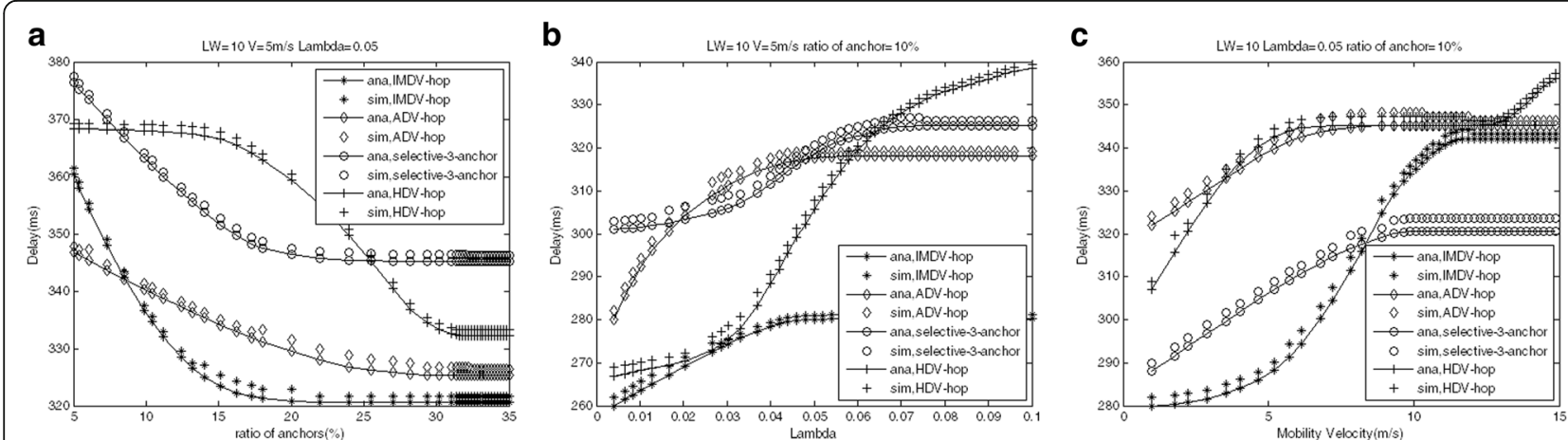

Fig. 11 Localization delay comparisons. a Localization delay comparisons for different schemes based on the ratio of anchors. b Localization delay comparisons for different schemes based on node density. c Localization delay comparisons for different schemes based on mobility velocity 

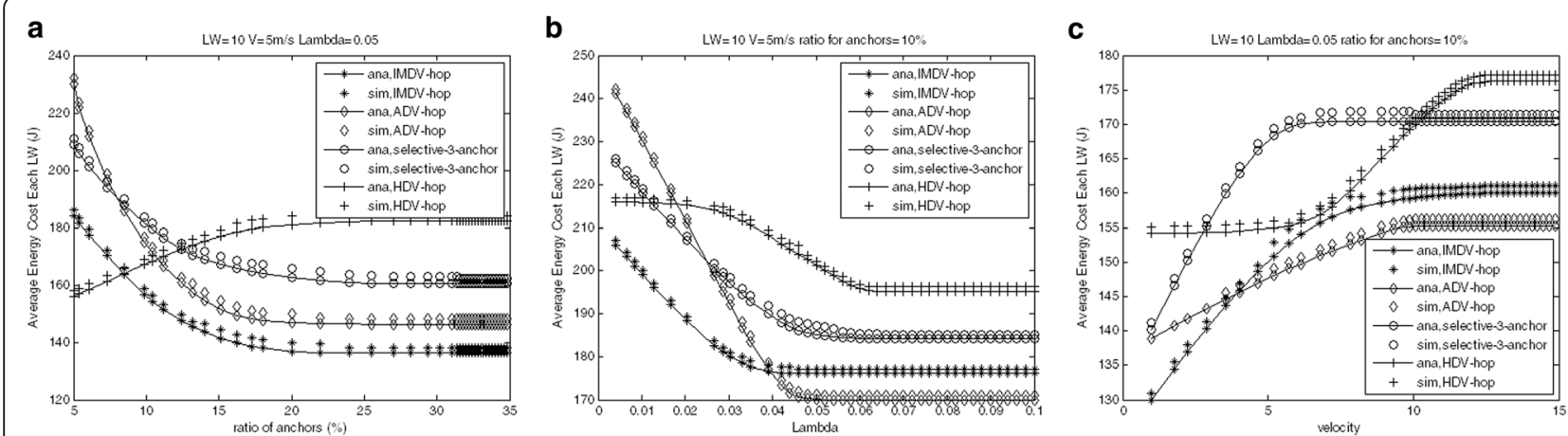

Fig. 12 Localization consumption comparisons. a Localization consumption comparisons for different schemes based on the ratio of anchors. b Localization consumption comparisons for different schemes based on node density. c Localization consumption comparisons for different schemes based on mobility velocity

velocity. Performance comparisons illustrated in Figs. 10, 11 and 12, IMDV-hop presents not fully up to expectations for relatively high velocity. But, it is noted that IMDV-hop is designed to collect circumstance parameter periodically and localize intermittent mobile aquaculture objects, and low velocity of $v \leq 8 \mathrm{~m} / \mathrm{s}$ is sufficient for our monitoring and localizing aquaculture networks.

Moreover, performance compares are also proposed between IMDV-hop and WMCL, which designs specially for mobile WSNs. In WMCL scheme, bounding-box is used to improve sampling efficiency by reducing the scope of selecting of candidate samples, accompanying with two-hop beacon neighbors' negative effects and sensor neighbors' estimated position information to reduce the bounding-box. Moreover, WMCL scheme can be also used for static localization, rather than be only used for mobility localization for other MCL schemes. But iterative seeking for candidate samples consume plenty of energy for WMCL scheme, and also, localization error of WMCL increases sharply when number of anchors decreasing.

Localization error increases in low velocity for WMCL scheme shown in Fig. 13a, such as $v \leq 6.15 \mathrm{~m} / \mathrm{s}$, and tends to steady values when the velocity is higher than $6.2 \mathrm{~m} / \mathrm{s}$. On the other hand, error for IMDV-hop increases smoothly in low velocity such as $v \leq 4.02 \mathrm{~m} / \mathrm{s}$, while increases sharply $v \geq 4.1 \mathrm{~m} / \mathrm{s}$, and error differentiation with WMCL scheme is growing bigger and bigger. Similarly, delay for IMDV-hop scheme presents the prior behavior when the velocity is lower than $v \leq 13.04 \mathrm{~m} / \mathrm{s}$ shown in Fig. 13b.

\section{Conclusions}

In this paper, we have presented range-free cross-layer localization scheme IMDV-hop based on DV-hop algorithm in intermittent mobile WSNs, accompanying HTC algorithm. At first, inferiorities of original DV-hop scheme are
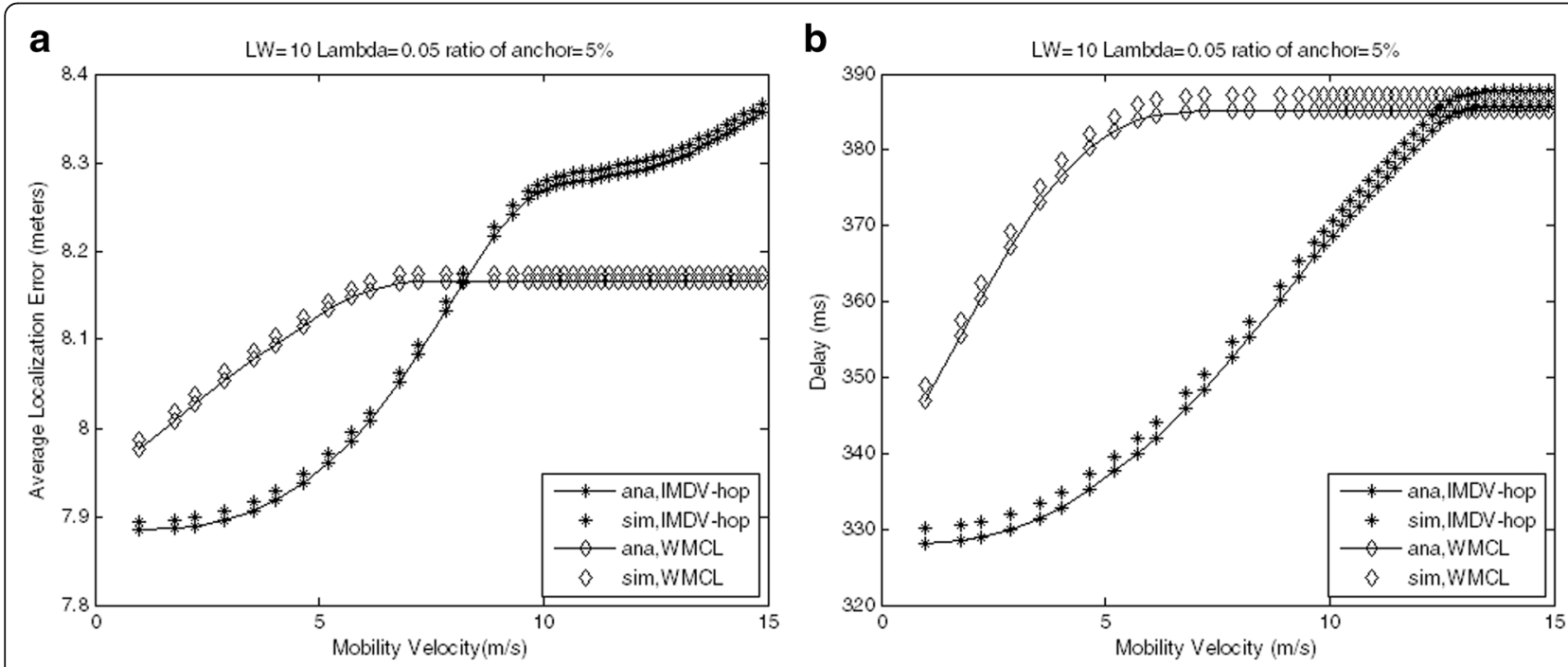

Fig. 13 a Localization error comparison based on mobility velocity. b Localization delay comparison based on mobility velocity 
denoted elaborately after brought briefly about it. Then, an improved localization scheme IMDV-hop embedded in WLS method is proposed, and two critical parameters, correction coefficient $k_{c}$ and weighted coefficient $w_{N_{x}, i}$, are introduced into it to improve localization performance. And then, localization performance is presented taking parameters describing about the network into account, such as anchor's number, node density, localization window, node's velocity, and transmission range. Moreover, comprehensive performance comparisons between IMDV-hop algorithm and other DV-hop-based schemes, between IMDV-hop and MCL-based scheme are proposed. Comprehensive NS-2 simulations according to these parameters demonstrate that analysis results of these models match well with simulation results, especially for lower mobile velocity and relative higher node density. Besides, analysis and comparison results show that delay behaviors of this low-velocity, time-critical scheme IMDV-hop is improved largely relative to other schemes, and localization accuracy is improved in some cases of more node density and lower mobile velocity.

For determining the location of nodes in mobile WSNs, anchor's ratio has significant impact on localization behaviors. But more anchors can bring out much energy and economic consumption. And if the propagation model is taken into account, the distance between unknown node and anchors plays greater role in localization performance. Moreover, mobility velocity of nodes including anchors and unknown nodes, either periodically or randomly moving, can also be questions in localization. In the recent future, we can also extend our analysis to these pending problems.

\section{Abbreviations}

ADV-hop: Advanced DV-hop; AOA: Angle of arrival; APIT: Approximate Point in Triangle; COMCL: Constraint rules Optimized Monte Carlo Localization Scheme; CPE: Convex position estimation; CSMA/CA: Carrier Sense Multiple Access with Collision Avoidance; DV-hop: Distance Vectorhop; GPS: Global Positioning System; HDV-hop: Hybrid DV-hop; HTC: Hidden Terminal Couple; IMCL: Improved MCL; IMDVhop: Intermittent Mobile DV-hop; IWC-DV-hop: Improved weighted centroid DV-hop; MCB: Monte Carlo localization boxed; MCL: Monte Carlo localization; NLLS: Non-linear least square; O-WSN: Hybrid optical WSN; RSSI: Received signal strength indicator; SMC: Sequential MCL; SSW: Sub-square weighted; TDOA: Time difference of arrival; TOA: Time of arrival; UWSNs: Underwater wireless sensor networks; WLS: Weighted least square; WMCL: Weighted MCL; WSN: Wireless sensor network

\section{Funding}

The experiments of this research are funded by the National Natural Science Foundation of China under grant number 61362017 and Building Foundation for Teaching Team of Shanghai Ocean University under grant number A1-0201-00-0322062.

\section{Authors' contributions}

JZ carried out the IMDV-hop localization scheme, accompanying with HTC transmission scheme, and helped to draft the manuscript. CLv carried out most of the simulation experiments. $\mathrm{ZT}$ carried out the defects of original DV-hop scheme and drafted the manuscript. All authors contributed to the work. All authors read and approved the final manuscript.

\section{Authors' information}

Jianping Zhu received Ph.D. degree in the Department of Electronic, Information and Electrical Engineering of Shanghai Jiaotong University, China. Currently, she is an associate professor of SOU College of Engineering Science and Technology in Shanghai Ocean University. Zhu's main research interests include wireless sensor networks, digital communication systems analysis and design, and information theory. Chunfeng Lv received Ph.D. degrees in the Department of Electronic, Information and Electrical Engineering of Shanghai Jiaotong University, China. Currently, he is a lecturer of SOU College of Engineering Science and Technology in Shanghai Ocean University. Lv's main research interests include detection technique using WSNs, digital communication systems analysis and design, and information theory. Zhengsu Tao received a Ph.D. degree in the Department of Electronics and Information Engineering from Hongkong University of Science \& Technology. Currently, he is a professor of the Department of Electronic, Information and Electrical Engineering of Shanghai Jiaotong University. His research interests include detection technique using WSNs.

\section{Competing interests}

The authors declare that they have no competing interests.

\section{Publisher's Note}

Springer Nature remains neutral with regard to jurisdictional claims in published maps and institutional affiliations.

\section{Author details}

${ }^{1}$ SOU College of Engineering Science and Technology, Shanghai Ocean University, No.999, Huchenghuan Rd, Shanghai, People's Republic of China. ${ }^{2}$ Department of Electronic, Information and Electrical Engineering, Shanghai Jiaotong University, No. 800, Dongchuan Road, Shanghai 200240, People's Republic of China.

Received: 20 January 2017 Accepted: 26 June 2018 Published online: 11 July 2018

References

1. J Aspnes, T Eren, DK Goldenberg, MA Stephen, W Whiteley, YR Yang, BDO Anderson, PNA Belhumeur, Theory of network localization. IEEE Trans. Mob. Comput. 5(12), 1663-1678 (2006)

2. Z Yang, YH Liu, Understanding node localizability of wireless ad hoc and sensor networks. IEEE Trans. Mob. Comput. 11(8), 1249-1260 (2012)

3. GQ Mao, B Fidan, BDO Anderson, Wireless sensor network localization techniques. Comput. Netw. 51, 2529-2553 (2007)

4. TJS Chowdhurya, C Elkina, D Devabhaktunia, DB Rawat, J Oluochc, Advances on localization techniques for wireless sensor networks: a survey. Comput. Netw. 110(9), 284-305 (2016)

5. HP Tan, R Diamant, WKG Seah, M Waldmeyer, A survey of techniques and challenges in underwater localization. Ocean Eng. 38, 1663-1676 (2011)

6. R Takemura, H Sakata, H Ishida, Active chemical sampling system for underwater chemical source localization. J. Sensors. 1, 1-11 (2016)

7. S Pace, G Frost, I Lachow, D Frelinger, D Fossum, DK Wassem, M Pinto. The global positioning system. (RAND Corporation, Santa Monica, 1995), pp. $237-270$

8. J Cheon, H Hwang, DS Kim, Y Jung, IEEE 802.15.4 ZigBee-based time-ofarrival estimation for wireless sensor networks. Sensors 16(2), 203 (2016)

9. KR Rao, TR Kumar, C Venkatnaryana, Selection of anchor nodes in time of arrival for localization in wireless sensor networks. Proceedings of the international conference on soft. Comput. Syst. 397, 45-57 (2016)

10. A Boukerche, HAB Oliveira, EF Nakamura, AAF Loureiro, Localization systems for wireless sensor networks. IEEE Wirel. Commun. 14(6), 6-12 (2007)

11. P Pivato, L Palopoli, D Petri, IEEE accuracy of RSS-based centroid localization algorithms in an indoor environment. IEEE Trans. Instrum. Meas. 60(10), 3451-3460 (2011)

12. F Gustafsson, F Gunnarsson, D Lindgren, Sensor models and localization algorithms for sensor networks based on received signal strength. EURASIP J. Wirel. Commun. Netw. 16, 1-13 (2012)

13. S Hamdoun, A Rachedi, A Benslimane, RSSI-based localisation algorithms using spatial diversity in wireless sensor networks. Int. J. Ad. Hoc. Ubiquitous. Comput. 19(3), 157-167 (2015) 
14. RC Jin, HX ZP Che, Z Wang, LD Wang, An RSSI-based localization algorithm for outliers suppression in wireless sensor networks. Wirel. Netw 21, 25612569 (2015)

15. D Niculescu, B Nath, Ad hoc positioning system (APS) using AOA. 22th annual joint conference of the IEEE computer and communications of IEEE societies. IEEE. For. Soc. 2003, 1734-1743 (2003)

16. R Stoleru, T He, JA Stankovic, Range-free localization. Secure localization and time synchronization for wireless sensor and Ad Hoc networks. Advances Inf. Secur. 30, 3-31 (2007)

17. SP Singh, SC Sharma, Range free localization techniques in wireless sensor networks: a review. Procedia Comput. Sci. 57, 7-16 (2015)

18. A Stanoeva, S Filiposkac, V In, L Kocarev, Cooperative method for wireless sensor network localization. Ad. Hoc. Netw. 40, 61-72 (2016)

19. TEren, Cooperative localization in wireless ad hoc and sensor networks using hybrid distance and bearing (angle of arrival) measurements. EURASIP J. Wirel. Commun. Netw. 72, 1-18 (2011)

20. J Heidemman, D Estrin, N Bulusu, GPS-less low cost outdoor localization for very small devices. IEEE Pers. Commun. Mag. 7(5), 28-34 (2000)

21. J Wang, P Urriza, UX Han, D Cabric, Weighted centroid localization algorithm: theoretical analysis and distributed implementation. IEEE Trans. Wirel. Commun. 10(10), 3403-3413 (2011)

22. C Laurendeau, M Barbeau, Centroid localization of uncooperative nodes in wireless networks using a relative span weighting method. EURASIP J. Wirel. Commun. Netw. 2010, 1-10 (2010)

23. L Doherty, KS Pister, L El Ghaoui, Convex position estimation in wireless sensor networks. Proceedings of IEEE twentieth annual joint conference of the IEEE computer and communications. For. Soc. 3, 1655-1663 (2001)

24. Z Liu, X Feng, TL JJ Zhang, YL Wang, An improved GPSR algorithm based on energy gradient and APIT grid. J. Sensors. 12, 1-7 (2015)

25. D Niculescu, B Nath, Ad hoc positioning system (APS). IEEE Conf. Glob. Telecommun. 5, 2926-2931 (2001)

26. D Niculescu, B Nath, DV based positioning in ad hoc networks. Telecommun. Syst. 22, 267-280 (2003)

27. H Chen, K SeZaki, P Deng, H CheungSo, An improved DV-hop localization algorithm with reduced node location error for WSNs. IEICE Trans. Fundam Electron. Commun. Comput. Sci. E91-A(8), 2232-2236 (2008)

28. A Boukerche, ABF Horacio, EF Nakamura, AF Antonio, DV-Loc: a scalable localization protocol using Voronoi diagrams for wireless sensor networks. IEEE Wirel. Commun. 16(2), 50-55 (2009)

29. SF Hou, XJ Zhou, XX Liu, A novel DV-hop localization algorithm for asymmetry distributed wireless sensor networks. 3rd IEEE International Conference on Computer Science and Information Technology. (Chengdu 2010), pp. 243-248

30. J Lee, WY Chung, E Kim, IW Hong, Robust DV-hop algorithm for localization in wireless sensor network. International Conference on Control, Automation and Systems. (Kintex, Gyeonggi-do, 2010), pp. 2506-2509

31. S Kumar, DK Lobiyal, An advanced DV-hop localization algorithm for wireless sensor networks. Wirel. Pers. Commun. 71, 1365-1385 (2013)

32. Y Hu, XM Li, An improvement of DV-hop localization algorithm for wireless sensor networks. Telecommun. Syst. 53, 13-18 (2013)

33. H Safa, A novel localization algorithm for large scale wireless sensor networks. Comput. Commun. 45, 32-46 (2014)

34. GZ Song, DY Tam, Two novel DV-hop localization algorithms for randomly deployed wireless sensor networks. Int. J. Dist. Sensor. Netw. 2015, 1-9 (2015)

35. S Tomic, I Mezei, Improvements of DV-hop localization algorithm for wireless sensor networks. Telecommun. Syst. 61, 93-106 (2016)

36. LQ Gui, T Val, A Wei, R Dalce, Improvement of range-free localization technology by a novel DV-hop protocol in wireless sensor networks. Ad Hoc Netw. 24, 55-73 (2015)

37. XY Li, LS Yan, W Pan, B Luo, Optimization of DV-hop localization algorithm in hybrid optical wireless sensor networks. J. Heuristics 21(2), 177-195 (2015)

38. GJ Han, J Chao, CY Zhang, L Shu, QW Li, The impacts of mobility models on DV-hop based localization in mobile wireless sensor networks. J. Netw. Comput. Appl. 42, 70-79 (2014)

39. X Chen, BL Zhang, Improved DV-hop node localization algorithm in wireless sensor networks. Int. J. Dist. Sensor. Netw. 2012, 1-7 (2012)

40. LX Hu, D Evans, Localization for mobile sensor networks. Proceeding MobiCom '04 Proceedings of the 10th annual international conference on Mobile computing and networking. (Philadelphia, 2004), pp. 45-57
41. S Thrun, D Fox, W Burgard, F Dellaert, Robust Monte Carlo localization for mobile robots. Artif. Intell. 128, 99-141 (2001)

42. A Baggio, K Langendoen, Monte Carlo localization for mobile wireless sensor networks. Ad Hoc Netw. 6(5), 718-733 (2008)

43. WKH JP Sheu, JC Lin, Distributed localization scheme for mobile sensor networks. IEEE Trans. Mob. Comput. 9(4), 516-526 (2010)

44. SG Zhang, JN Cao, LJ Chen, DX Chen, Accurate and energy-efficient rangefree localization for mobile sensor networks. IEEE Trans. Mob. Comput. 9(6), 897-910 (2010)

45. Z Wang, YL Wang, MD Ma, W JG, Efficient localization for mobile sensor networks based on constraint rules optimized Monte Carlo method. Comput. Netw. 57(14), 2788-2801 (2013)

46. H Rashid, AK Turuk, Dead reckoning localisation technique for mobile wireless sensor networks. IET Wireless. Sensor. Syst. 5(2), 87-96 (2015)

47. S Halder, A Ghosal, A survey on mobility-assisted localization techniques in wireless sensor networks. J. Netw. Comput. Appl. 60, 82-94 (2016)

48. RB M Qin, A Zhu, Monte Carlo localization method based on differential evolution optimization applied into economic forecasting in mobile wireless sensor networks. EURASIP J. Wirel. Commun. Netw. 1-9, 32 (2018)

49. JP Zhu, CF Lv, ZS Tao, Performance analyses and improvements for IEEE 802.15.4 CSMA/CA scheme in wireless multi-hop sensor networks based on HTC algorithm. Int. J. Dist. Sensor Netw. 2013, 1-21 (2013)

50. J Li, JM Zhang, XD Liu, A weighted DV-hop localization scheme for wireless sensor networks. IEEE Int. Conf. Scalable. Comput. Commun. 2009, 269-272 (2009)

51. ML Jiang, JY Luo, XK Zou, Research on algorithm of three-dimensional wireless sensor networks node localization. J. Sensors. 5, 1-9 (2016)

\section{Submit your manuscript to a SpringerOpen ${ }^{\circ}$ journal and benefit from:}

- Convenient online submission

- Rigorous peer review

- Open access: articles freely available online

- High visibility within the field

- Retaining the copyright to your article

Submit your next manuscript at $>$ springeropen.com 